\title{
Eye Position Effects on Visual, Memory, and Saccade-Related Activity in Areas LIP and 7a of Macaque
}

\author{
Richard A. Andersen, ${ }^{1}$ R. Martyn Bracewell, ${ }^{1}$ Shabtai Barash, ${ }^{1}$ James W. Gnadt, ${ }^{2}$ and Leonardo Fogassi ${ }^{1,3}$ \\ 'Department of Brain and Cognitive Sciences, Massachusetts Institute of Technology, Cambridge, Massachusetts 02139, \\ 'Department of Physiological Optics, University of Alabama, Birmingham, Alabama 35294, and ${ }^{3}$ Istituto di Fisiologica \\ Umana, 43100, Parma, Italy
}

\begin{abstract}
We studied the effect of eye position on the light-sensitive, memory, and saccade-related activities of neurons of the lateral intraparietal area and area $7 \mathrm{a}$ in the posterior parietal cortex of rhesus monkeys. A majority of the cells showed significant effects of eye position, for each of the 3 types of response. The direction tuning of the light-sensitive, memory and saccade responses did not change with eye position but the magnitude of the response did. Since previous work showed a similar effect for the light-sensitive response of area 7a neurons (Andersen and Mountcastle, 1983; Andersen et al., 1985b), the present results indicate that this modulating effect of eye position may be a general one, as it is found in 3 types of responses in 2 cortical areas. Gain fields were mapped by measuring the effect of eye position on the magnitude of the response at 9 different eye positions for each neuron. The gain fields were usually planar or largely planar for all 3 types of response in both areas, indicating that the magnitude of the response usually varies linearly with both horizontal and vertical eye position. A similar observation was made previously for the gain fields of the lightsensitive response of area $7 \mathrm{a}$ neurons (Andersen et al., 1985b). Although gain fields sloped in all directions for the population of cells, the gain field slopes of the light-sensitive, memory and saccade responses for individual cells were usually similar. It is proposed that these eye position effects play an important role in making coordinate transformations for visually guided movement.
\end{abstract}

One of the fundamental problems in programming movements under visual guidance is how to link the inherently different coordinate systems used at the input and output stages. The visual inputs are derived from images on the retinas and are cast in retinal coordinates. Due to a point-to-point mapping at the lower levels of the visual pathway, this retinotopic representation is maintained in many brain structures. On the other hand, movements must be made to locations in space with

\footnotetext{
Received June 16, 1989; revised Sept. 5, 1989; accepted Sept. 28, 1989.

This work was supported by NIH grant EY05522, ONR contract N00014-89J1236, the Sloan Foundation, and the Whitaker Health Sciences Foundation. R.M.B. was supported in part by a Surdna predoctoral fellowship. We thank G. Robertson, R. Keough, and S. Marchetti for technical assistance, and C. Andersen and C. Cooper for editorial assistance.

Correspondence should be addressed to Richard A. Andersen, Department of Brain and Cognitive Sciences, Massachusetts Institute of Technology, E25-236, Cambridge, MA 02139

Copyright (C) 1990 Society for Neuroscience $0270-6474 / 90 / 041176-21 \$ 02.00 / 0$
}

respect to the body, and therefore, the motor outputs must encode movements in body-centered coordinates.

A brain area likely to accomplish these coordinate transformations is the posterior parictal cortex. The posterior parictal cortex of both monkeys and humans has been implicated through lesion studies as playing a crucial role in spatial perception and movement under visual guidance (see Andersen, 1987, for review). In particular, human patients with posterior parietal lesions exhibit misreaching to visual locations despite having no overt visual field defects. Similar results are seen in monkeys and suggest that the deficit is a result of coordinate transformation disruption rather than a primary perception defect (see Andersen, 1987, for review). Interestingly, lesions to the posterior parietal cortex produce deficits that are specific to saccades made to visual targets and not to saccades made on verbal command or to auditory or somatosensory stimuli, confirming that this is a visual area (see Andersen and Gnadt, 1989, for review).

In this article we focus on coordinate transformations for visually guided saccadic eye movements in rhesus monkeys. The saccade system is simpler than other motor systems, including those involved in moving the limbs, and therefore is easier to study. The number of muscles involved is fewer; there is a constant load or inertia for the eyeball; and the eye and orbit can be considered a single joint. The coordinate transformation problem remains fundamentally the same, however: visual targets for saccades are provided in retinal coordinates, while the motor commands must specify the desired location of the eyes in the orbits.

Recently we have described a new area in the posterior parietal cortex buried in the lateral bank of the intraparietal sulcus, the lateral intraparietal area (LIP), which appears to play a role in saccadic eye movements. This area was originally described on anatomical grounds, as it has much stronger connections to saccade centers, including the frontal eye fields and superior colliculus, than other areas in the posterior parietal cortex (Andersen et al., 1985a). Functional recording experiments in area LIP indicate that the cells respond to visual stimuli, saccades and eye position; often all 3 types of response are found in single cells (Gnadt and Andersen, 1988). Although adjoining parietal area $7 \mathrm{a}$ contains neurons that also have saccade-related responses (Lynch et al., 1977; Andersen et al., 1987), many more of the saccade-related responses in area LIP are presaccadic (Andersen and Gnadt, 1989; Barash et al., 1988) and the visual and memory (M) responses for the same visual stimulus are larger, on average, evoking more action potentials in the re- 
sponse. Memory-related activities have also been noted in the prefrontal cortex (Goldman-Rakic, 1988), frontal eye fields (Bruce and Goldberg, 1985) and superior colliculus (Mays and Sparks, 1980); all these areas are connected to area LIP.

In previous studies in our laboratory we found that eye position and retinal position signals in area $7 \mathrm{a}$ interacted in a manner appropriate for encoding the location of visual stimuli in head-centered coordinates (Andersen et al., 1985b; Andersen and Zipser, 1988). In particular, although the interactions between the 2 signals were nonlinear and complex, 2 general consistencies were identified in the data. The first observation was that while the visual receptive fields remained retinotopic, the evoked visual response was modulated by eye position. The second observation was that the effect of eye position on the total response of the cell varied linearly as a function of both horizontal and vertical eye position for retinotopically identical stimuli. The plots of the effect of varying eye position, with constant retinal position, were referred to as gain fields, and a majority of area 7a gain fields were found to be planar or to have significant planar components. In computational studies it was found that neural network models trained to transform eye position and retinal position inputs, such as those converging on area $7 \mathrm{a}$, to output locations in head-centered coordinates produced middle-layer units with features similar to those found in area 7a (Andersen and Zipser, 1988; Zipser and Andersen, 1988). These model units exhibited similar planar gain fields, with modulation of retinal receptive ficlds by cyc position, to those found in the neural data described above. These computational studies raise the interesting possibility that the model may employ an algorithm similar to that used by the brain for transforming coordinates in a parallel network architecture.

In the present experiments we directly compare, in the same animals, the effects of eye position on the visual, memory, and saccade responses in areas LIP and $7 a$. We confirm the results previously recorded for eye position effects on the visual responses of area 7 a cells and find that the same effects operate for all 3 responses in both cortical fields. These results suggest that area LIP may play a role in processing the coordinate transformations required for saccadic eye movements. They also suggest that the same algorithm for coordinate transformation may be used by both areas $7 \mathrm{a}$ and LIP.

\section{Materials and Methods}

Single cell recordings were made in awake, behaving monkeys trained in eye movement tasks. Recordings were made from 3 hemispheres of 2 rhesus monkeys. Eye position was measured by the scleral search coil technique, which involves recording the induced current in a coil, surgically implanted on the sclera of the eye, as it moves in a magnetic field (Fuchs and Robinson, 1966; Judge et al., 1980).

The experiments were performed in a light-tight chamber, and the animal was monitored with a remote infrared camera and infrared light source. The visual stimuli and fixation point were points of light $0.5^{\circ}$ in diameter, back-projected on a large projection screen. The tangent screen was located $57 \mathrm{~cm}$ from the animal's eyes. Three projectors, with electronic shutters and $\mathrm{x}$, $\mathrm{y}$ galvanometers, were under computer control and were used to project the visual stimuli and fixation point. Stimulus intensities were 45 candella $/ \mathrm{m}^{2}$ against a totally dark background. Optical screens under computer control were used to adjust the luminescence of the stimuli so that they were always of the same value, measured at the eye of the monkey, independent of screen location. Since it was found for monkey M13 that the small changes in luminescence with screen location had no effect on the activity of the 7a and LIP neurons, this control was not used for monkey M33. The computer corrected for screen tangent errors to ensure that visual stimuli could be positioned at identical retinal eccentricities regardless of eye position.
Memory-saccade task. In the basic paradigm the animal was required to make eye movements from different orbital positions to the remembered location in the dark. The change in visual, memory and saccade activity for equal-amplitude eye movements was measured in two different cortical fields. The task was designed to test for visual, memory and saccade responses in one trial. Additionally, it was used to rule out possible visual artifacts contributing to the apparent eye position effects on the saccade response. Such artifacts could arise either from the onset of a visual target that triggers eye movement or from movement in contours of the visual background through the cells' receptive fields during the eye movement.

The animals were trained to make saccades to remembered locations in total darkness. Figure 1 illustrates the task: after a randomly interleaved intertrial interval of 500-1500 msec, a fixation light appeared at the location on the screen corresponding to straight-ahead fixation $(0$, $0)$. "Straight ahead" is defined by a line perpendicular to the coronal plane of the monkey, intersecting the plane at a position equidistant between the 2 eyes. The animal had $1 \mathrm{sec}$ to fixate the stimulus, for the trial to begin. Fixation was indicated by the eye position recordings and was monitored by the computer, which required the eye to be fixated on the fixation point for a continuous period of $100 \mathrm{msec}$ before the trial could begin. If the animal did not fixate the stimulus, a "miss" was recorded and the trial sequence was presented again after a new intertrial interval. If it did fixate, after either $300 \mathrm{msec}(23 \%$ of the cells) or $800 \mathrm{msec}(77 \%$ of the cells), a second stimulus flashed pseudorandomly at 1 of 8 locations at angle increments of $45^{\circ}$. The screen locations of the targets were located on a square grid at $(-15,15),(0,15),(15$, $15),(-15,0),(15,0),(-15,-15),(0,-15)$, and $(15,-15)$ measured in degrees of visual angle. Since the diagonal saccades were slightly longer, for 15 neurons the saccade targets were presented on both a circle and a square. Although the amplitudes were all identical on the circle, no significant difference in direction tuning between the circular and square patterns was noted. This result is likely due to the fact that the amplitude tuning of area LIP and 7 a saccade responses are broad, and the visual receptive fields are very large. The stimulus was present for only $300 \mathrm{msec}$, after which it was extinguished and the animal was required to remember its spatial location. Following a $400-\mathrm{msec}(33 \%$ of the cells) or $700-\mathrm{msec}$ ( $67 \%$ of the cells) delay the initial fixation light also went off, which commanded the animal to make an eye movement to the remembered location of the flashed target. If the animal broke fixation during the waiting period, the trial was registered an "error," the animal did not receive a reward, and the trial sequence began again. After the fixation point went off, the animal had $500 \mathrm{msec}$ to fixate the remembered target location. If the animal succeeded and maintained steady fixation at the remembered location for $500 \mathrm{msec}$, it received a drop of juice as reward.

The memory saccade task ensured that the saccade response did not result either from the target, since it had been removed for a long period before the saccade, or from the visual background, since the saccade was made in total darkness. To ensure that the response did not result from the offset of the fixation point, 1 of the 9 classes of each run consisted of a control, shown in Figure $1 B$. In this control, no saccade target is presented and the animal's task is to maintain fixation at the fixation point while it is extinguished for $500 \mathrm{msec}$. No cells in this analysis showed any response to the offset of the fixation target and all are similar to the one in Figure $1 B$.

The electronic windows for maintaining all fixation positions were $\pm 10^{\circ}$ in animal M13 and a range of widths, usually from \pm 1.5 to $15^{\circ}$, in animal M33. If the animal's eye did not move to gaze within the window of space centered on the test location, the trial was an error. In $\mathrm{M} 33, \pm 1.5^{\circ}$ windows were used for saccades to visual targets and the larger windows were used for the memory saccades. Such large windows were used in the memory task because there is a systematic distortion of eye movements to remembered targets for both macaques and humans, with upward saccades typically being hypermetric and downward saccades typically hypometric (Gnadt et al., 1987, 1989). We have not found it possible to reduce this distortion even after training an animal on smaller windows for several months (Gnadt et al., 1987, 1989). In animal M33 we generally used $\pm 7.5, \pm 10, \pm 12.5$, or $\pm 15^{\circ}$ windows, depending on the difficulty of the task. Only rarely did we use the $\pm 15^{\circ}$ window, and the animal did not adopt a strategy of not making an eye movement to the target, probably because this window size was used infrequently.

Training. Under general anesthesia and sterile surgical conditions, an acrylic skullcap for immobilizing the head and an eye coil for eye po- 


\section{A) Memory Saccade Task}

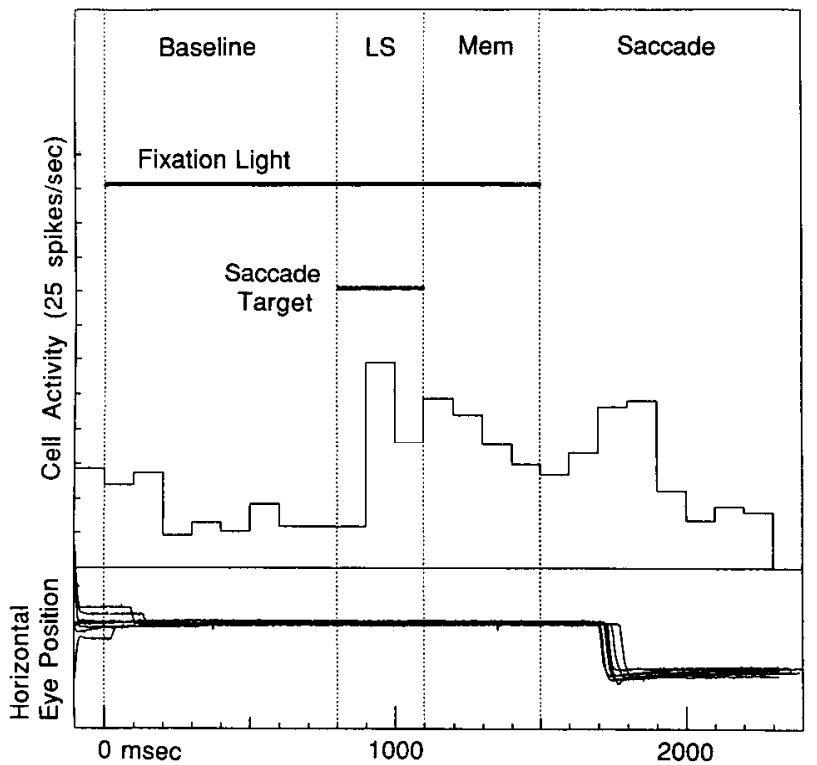

C) Saccade Direction Test
B) Control Task

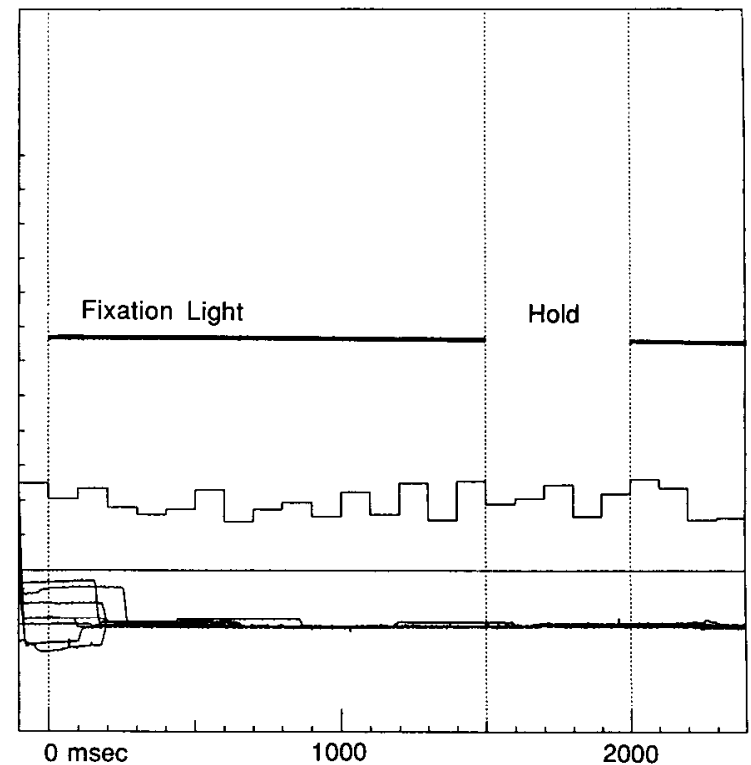

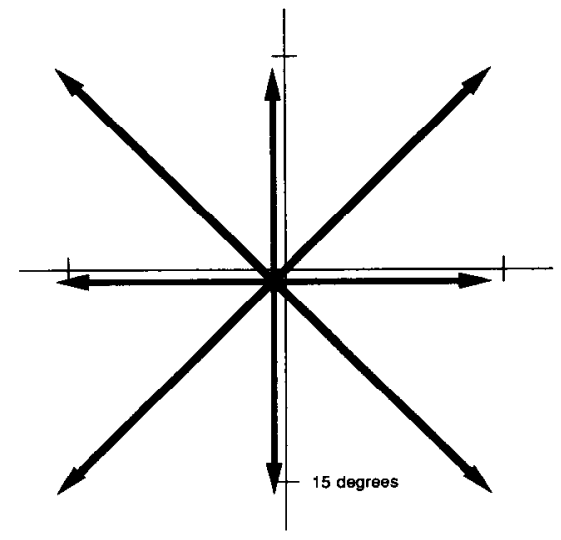

D) Gain Field Test

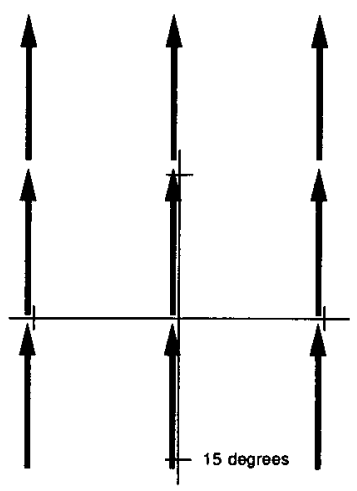

Figure 1. A, Memory saccade task showing the sequence of events. The baseline period represents the time prior to the onset of the fixation light and was always $800 \mathrm{msec}$ long. The saccade target appeared next for $300 \mathrm{msec}$ followed by a 400 - or 700-msec delay (memory period; a 400 -msec period is illustrated). Following the memory period, the fixation light is turned off commanding the animal to make a saccade to the remembered location of the saccade target in total darkness. The histogram shows activity from an area LIP neuron during the baseline, light sensitive (LS), memory (mem) and saccade periods. Firing rates were determined during these epochs; the method of measurement of spike rate is detailed in the text. Below the histogram are traces of the horizontal eye position indicating the time at which the animal made a saccade $15^{\circ}$ to the left. For horizontal eye position traces in all figures, down corresponds to leftward eye movements and up to rightward eye movements. $B$, Control task to show that the saccade response was not a result of the offset of the fixation target. The fixation light goes off for $500 \mathrm{msec}$ beginning at $1500 \mathrm{msec}$ after the initiation of the trial. The animal is trained not to make an eye movement if no saccade target is presented prior to the offset of the fixation point. The histogram indicates that the cell does not respond to the offset of the fixation target and the eye trace indicates that the animal did not make an eye movement during the holding period when the fixation point is extinguished. $C$, Task that tests for the direction selectivity of the light sensitive, memory, and saccade responses. Each computer run consisted of 8 classes of memory saccades, each calling for an eye movement in a different direction, and a control class (illustrated in $B$ ); the 9 classes were presented in random block design for 8-10 trials in each class. The axes indicate screen coordinates in degrees of visual angle. $D$, Test for determining the gain fields. Once the best direction of a neuron has been determined by the test in $C$, memory saccades are made in that best direction, but from 9 different initial eye positions.

sition recording were implanted. Training began a week after surgery and initially consisted of having the animal learn to fixate the fixation target with the head fixed. Next the animal learned to fixate at different orbital positions and to saccade to the onset of a saccade target that occurred simultaneously with the offset of the fixation target. Finally, the animal was trained to withhold the response for longer periods as described in the memory saccade task outlined above.

Recording. Once training was complete, a second surgery was performed in which a recording chamber was mounted over Brodmann's areas 5 and 7 in the posterior parietal cortex. Electrode penetrations 
were made into area $7 \mathrm{a}$ on the gyral surface of the inferior parietal lobule and either down the bank of the intraparietal sulcus into area LIP or through area 5 into area LIP. Before each daily experimental session, the eye movement recording system was calibrated by having the animal fixate 9 positions each separated by $20^{\circ}$ on a grid array centered on straight-ahead fixation. Slopes and intercepts for horizontal and vertical eye position were generated in digital-to-analogue conversion (DAC) units from the calibration trials and were entered into the data collection program for setting the eye position windows. Recording sessions typically lasted up to $6 \mathrm{hr}$ daily including rest periods, and typically 3-5 cells were isolated and studied in detail on a given day.

When a cell was isolated, the direction tuning was measured using the memory saccade task from orbital position $(0,0)$ (Fig. 1C). Eight directions and the no-movement control were presented as 9 classes in a random block design and 8-10 responses were collected for each class (Fig. 1C). The results of this test determined the best directions of the visual, memory, and saccade responses (greatest neuronal response measured in number of action potentials). The 3 responses generally had the same best-direction tuning (Barash et al., 1989).

The next, and most crucial, test consisted of presenting the memory task in the best direction of the cell, but from 9 different orbital positions (see Figs. $1 D$ and 5 ). In each case a $15^{\circ}$ saccade was made (in the best direction) from 9 different orbital positions spaced $15^{\circ}$ apart on a $3 \times$ 3 grid centered on 0,0 . This test determined the effect of eye position on the visual, memory and saccade responses for the best direction of the neuron. The result of this test, which varies initial eye position while maintaining all other parameters constant, is referred to as a gain field.

If we were able to maintain recording for long enough periods, additional tasks were administered. For many cells the gain field mapping experiment was repeated for other memory-saccade directions. In some cells we tested all 8 directions at the best and worst eye positions. In many cells we were able to measure the eye position activity alone by requiring the animal to fixate for $2.5 \mathrm{sec}$ at 9 different locations on the screen (Fig. 3). The eye-position-only task used a $3 \times 3$ grid with $15^{\circ}$ spacings centered on $(0,0)$. In some cells the visual field was mapped by having the animal maintain fixation at a fixation target while ignoring stimuli flashed at different locations in the visual field.

Histology. In the last few weeks of the experiment involving monkey M13, lesions were made at the end of penetrations in both hemispheres by passing small direct current through the recording electrode at different depths along the electrode tract. $\Lambda t$ the conclusion of the experiments Ml3 was given an overdose of sodium pentobarbital and then perfused transcardially with heparinized saline. Guide wires were lowered into the brain at selected chamber coordinates immediately after the animal was killed. The wires were used as landmarks for blocking the posterior parietal cortex and for determining the approximate locations of recording tracks that were made early in the recordings. Lesions were not made at the early recording sites since typically the lesions are visible only for about 6 weeks after they are made. Good agreement was found between the locations of the guide wires and the coordinates of the actual lesions, indicating that the early recording locations, predicted from the coordinate system of the microdrive, appeared to be accurate.

Sections were made at $30-\mu \mathrm{m}$ thickness with sections stained alternately within thionin for cytoarchitecture and with the Gallyas method for myeloarchitecture (Gallyas, 1979).

For monkey M13, area LIP was identified by myeloarchitectural and physiological criteria. The majority of area LIP is identified by the densely myelinated area on the posterior aspect of the lateral bank of the intraparietal sulcus. This densely myelinated region is approximately $10 \mathrm{~mm}$ long in the anterior-posterior dimension of the sulcus and $3-4$ mm wide in the dorsal-ventral axis (Ungerleider and Desimone, 1986; Andersen et al., 1989). Ungerleider and Desimone (1986) have referred to this densely myelinated zone as VIP*. Area LIP continues 1-2 mm dorsal to the densely myelinated area (Gnadt and Andersen, 1988; Barash et al., 1989; Blatt et al., 1989) and was identified on physiological grounds. Area LIP neurons have much brisker responses during both the visual stimulus and the delay period and generally have shorter latency saccade responses (Andersen and Gnadt, 1989; Barash et al., 1989) than 7a neurons. Although there is local grouping of receptive fields according to retinotopic location, the overall organization of area LIP is complex and does not conform to a simple, continuous retinotopic map (Blatt et al., 1987, 1989). Only one hemisphere was recorded from in monkey 33 and this animal is presently being employed in ongoing experiments. The location of area LIP was determined in this animal based on physiological criteria and the recording depths in the sulcus.
Data analysis. Activity printouts of the gain fields were made for each cell and were used to choose time windows for the data analysis. Windows for the analysis of firing frequency were placed around different periods in the task. In a typical trial the visual stimulus was delivered at $800 \mathrm{msec}$ for a duration of $300 \mathrm{msec}$. Memory periods after the offset of the target were 400 or $700 \mathrm{msec}$ at which time the fixation light turned off, initiating the saccade. Typical windows for the data analysis were 300-800 msec for the background activity measured at the beginning of the trial, $900-1200 \mathrm{msec}$ for the visual response, $1200-1500 \mathrm{msec}$ in the 400-msec waiting period, and $1300-1800 \mathrm{msec}$ for the $700-\mathrm{msec}$ waiting period. Saccade windows were typically $1600-1900$ for the 400 msec waiting period trials and $1900-2200$ for the $700-\mathrm{msec}$ trials. Windows were adjusted slightly on a cell-by-cell basis to take into account differences in response latency or duration. Care was taken to ensure that the memory period did not include the activity peak associated with the visual response, which fell off rapidly after the offset of the visual target. In tests in which the visual stimulus used to map visual fields was the same as the saccade target, and in which the animal was trained not to saccade to the target, the visual response typically decayed to baseline within $100 \mathrm{msec}$. Only one set of windows was used on all 9 classes of each gain field test.

The activity rates for the visual, memory and saccade periods were computed both as total activity during the time period and with the background rate subtracted from the total rate. The background rate was typically computed from the time window spanning 300-800 msec from the beginning of the trial, i.e., before the onset of the saccade target. For the saccade response the background rate was measured during the memory period just before the saccade, rather than at the beginning of the trial, since we were interested in the increase in activity that occurred above the memory response. Occasionally there was a late saccade response and the memory response continued through what would normally be the saccade period. The saccade response appears to add to the memory response, which is why we use the period just before the saccade as the background measure. An analysis program extracted the action potentials that occurred during the chosen time periods, computed the average frequency of firing for each of these periods, and produced a file with these values for statistical analysis.

The statistical analysis used conventional linear regression techniques to partition the variability into components dependent on $X$ and $Y$ eye positions and residual "pure error" and "lack-of-fit" components for statistical testing (Kleinbaum and Kupper, 1978; Netter and Wasserman, 1983). The effects of horizontal and vertical eye position were assumed to be additive and noninteracting. Since all observations for each neuron fell into 9 groups, estimates of pure error variability are comparable to "within group" variability and were obtained in order to determine goodness-of-fit to the planar model.

The sum-of-squares lack-of-fit was calculated as the difference between the sum-of-squares of the model data and the sum-of-squares of the pure error. The $F$ ratio was computed from the ratio of mean-square lack-of-fit divided by the mean-square pure error. In other words, the error of the model should be approximately equal to the pure error if the planar model is valid. If the model error is significantly greater than the pure error, the planar model is not the best model to fit the data In order to determine whether there was a significant planar component to the data, an $r^{2}$ value (ratio of explained variation to total variation) was computed and used to test the significance of the sample correlation coefficient as an estimate of the population correlation coefficient. Coefficients for horizontal and vertical slope and $Z$-axis intercept (the intercept of the plane on the $Z$ axis) were computed as part of the regression analysis. The direction of the gradient of the planar component is defined as the direction of greatest positive slope and was computed by taking the inverse tangent of the ratio of the vertical and horizontal slopes of the plane. The best direction of the light-sensitive, memory, and saccade responses was determined by computing the direction of the axis of symmetry for the response. The axis of symmetry was the direction of a regression line through a polar plot of the responses that gave the smallest value for the sum of the squares of the differences between the line and the data points (Fig. 3).

\section{Results}

\section{Data base}

Rccordings were made from 3 hemispheres of 2 rhesus monkeys. A total of 409 cells were studied in quantitative experiments 


\section{A) $200 \mathrm{msec}$ Delay}

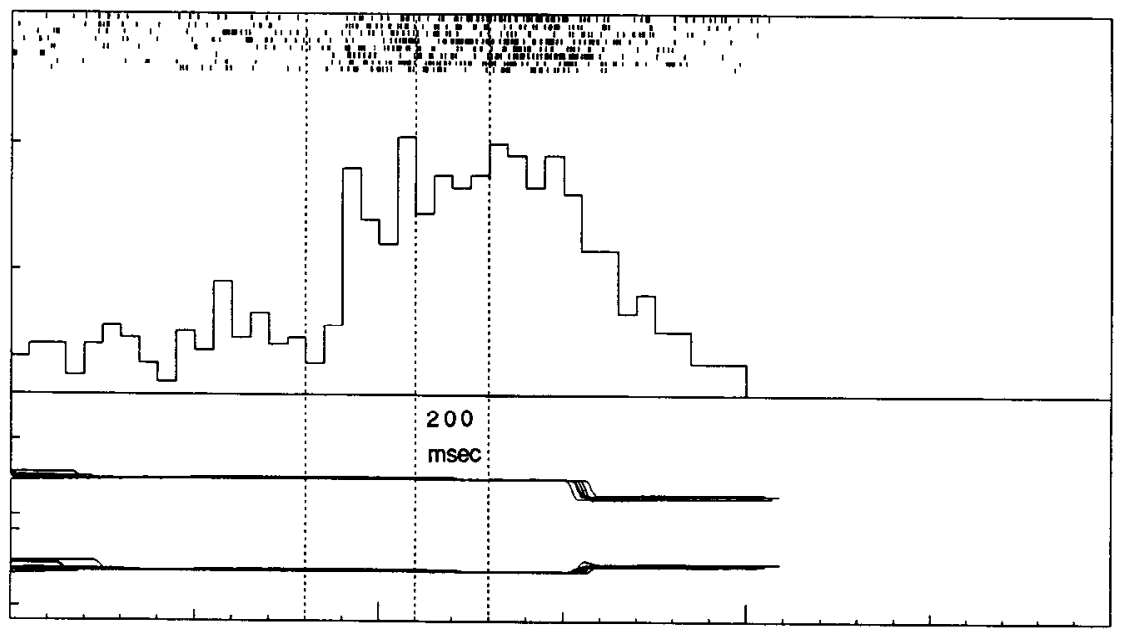

\section{B) $1000 \mathrm{msec}$ Delay}

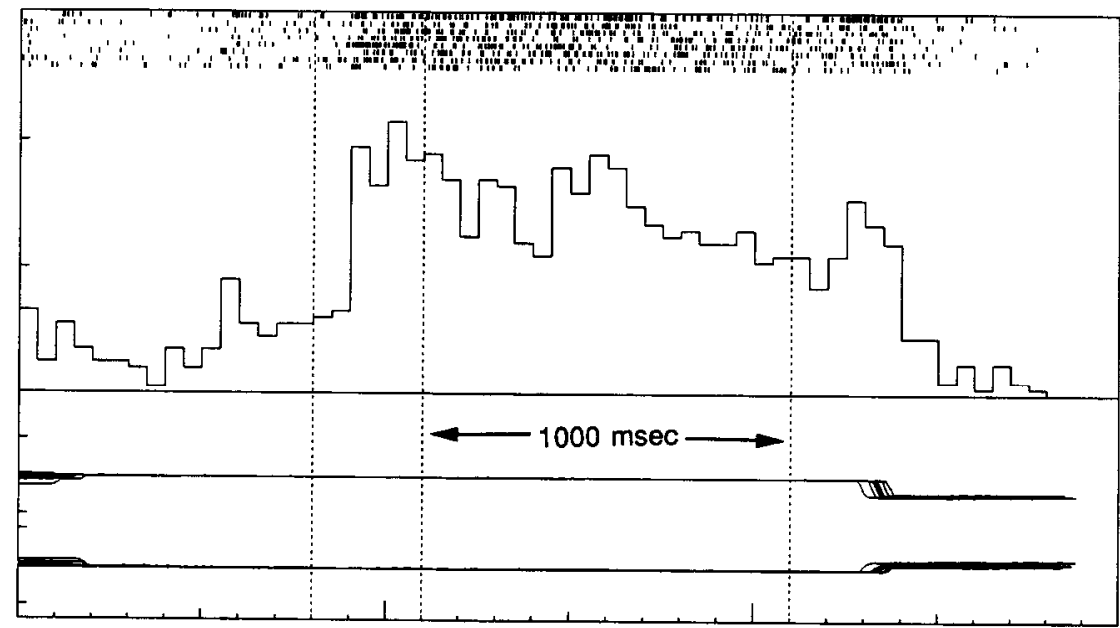

Figure 2. Memory saccade task with different delays demonstrating the memory character of the activity during the delay. Delays are $200 \mathrm{msec}(A), 1000$ $\mathrm{msec}(B)$, and $1300 \mathrm{msec}(C)$. The rasters show the actual neural activity that is used to make the histograms. The period between the first 2 dotted vertical lines represents the time the saccade target is present and the period between the second and third lines is the delay period. The fixation light goes off coincident with the third dotted vertical line. Both horizontal $(H)$ and vertical $(V)$ eye position traces are shown. In this experiment the saccade target appeared $15^{\circ}$ to the left. There is a vertical component in the leftward eye movement; this upward component for horizontal eye movements is common for saccades to remembered locations made in the dark (see Gnadt et al., 1987, 1989).

\section{C) $1300 \mathrm{msec}$ Delay}

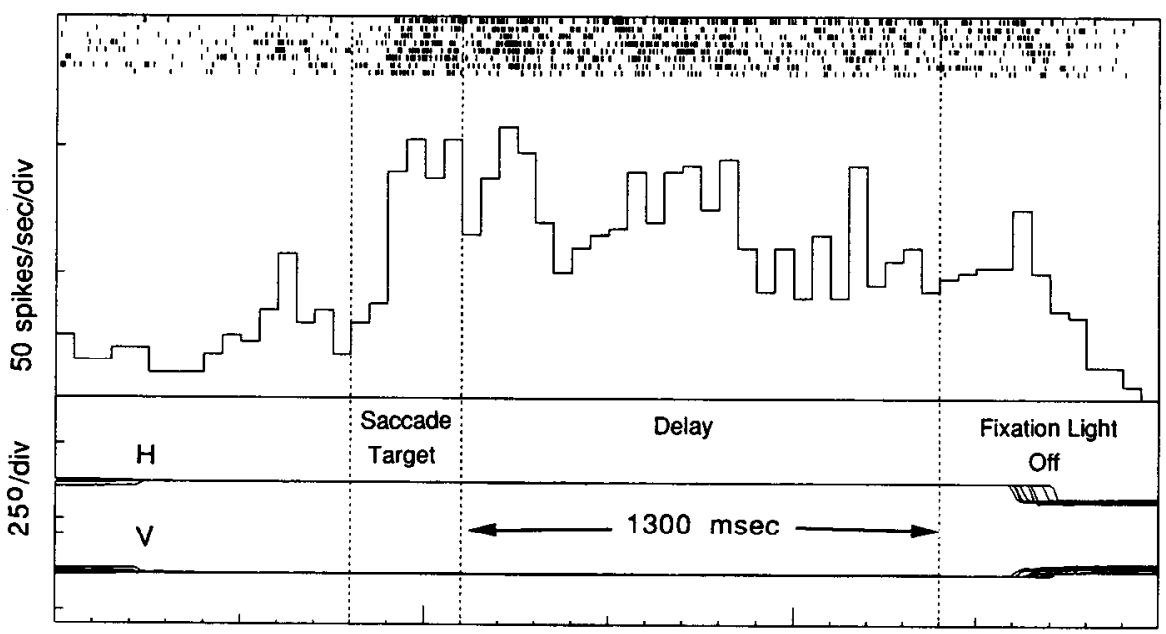

$100 \mathrm{msecs} / \mathrm{div}$ 


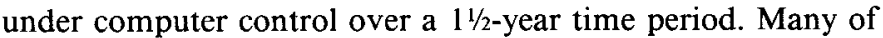
these cells were also used to examine saccade parameters and these data will be reported in subsequent papers (Barash et al., $1988,1989)$. For 126 cells, eye position effects on visual, memory and saccade responses were measured. However, in 35 of these neurons only 5 eye positions were studied and these cells subsequently were not used for more detailed statistical analysis. The 91 remaining cells had gain fields mapped at 9 eye positions and these cells form the basis of this study. Thirty of these cells were recorded from monkey M13 and 61 cells were recorded from monkey M33. For monkey M13, 21 cells were recorded from area LIP and 11 from area 7a, and for monkey M33, 34 cells were from area LIP and 25 from area $7 \mathrm{a}$. In all, 55 neurons of the sample were from area LIP and 36 were from area 7a. No major differences in eye position effects on the visual, memory, or saccade responses were seen for the 2 cortical fields. Most area 7a saccade responses were postsaccadic $(83 \%)$ whereas over half $(59 \%)$ of the area LIP responses were presaccadic (Barash et al., 1989). Also, area LIP memory, saccade, and visual responses were stronger than those recorded for area 7 a (Barash et al., 1989).

\section{Responses of area LIP and 7 a neurons}

Neurons from areas LIP and 7a exhibited 3 types of activity in the memory saccade paradigm: light-sensitive(LS), memory (M) and saccade (S) related responses. Figure $1 A$ is a typical example of activity, in this case for an area LIP neuron. There is a visual response that begins after the onset of the stimulus, then prolonged activity in the delay period, and finally a second peak of activity occurring at the time of the saccade. Since the saccade is made in total darkness the saccade-related response cannot be an artifact of visual stimulation. For 12 neurons, tests were made in both a lighted $\left(1\right.$ candella $\left./ \mathrm{m}^{2}\right)$ and dark test chamber. No appreciable differences were found in the responses under these 2 conditions.

To control for the possible artifact that the response could be related to the offset of the fixation point, a control class was used. In this control class, illustrated in Figure $1 B$, no saccade target is flashed in the visual field before the offset of the fixation target. The animal has been trained not to make an eye movement if no target is given, but rather to maintain fixation at the remembered location of the fixation target. The target reappears $500 \mathrm{msec}$ later. The eye position trace in Figure $1 B$ shows that the animal did not move his eyes and the spike histogram indicates that there was no response to the offset of the fixation point. No cells used in this study showed any response to the fixation target offset. A second indication that the response is not an artifact of fixation target offset is the observation that the saccade responses are almost always direction tuned; a simple offset response would not depend on the direction of the programmed eye movement.

Figure 2 shows why the activity in the delay period is believed to be memory-related. The response continues as long as the animal withholds its saccade response. In other experiments we have shown that this memory activity is coded in motor coordinates and therefore represents the intent of the animal to make an eye movement of a particular direction and amplitude (Gnadt and Andersen, 1986, 1988).

Figure 3 shows an example of direction tuning for LS, M, and $\mathrm{S}$ activity in an area LIP neuron. Note that all 3 responses are greatest when the animal saccades up. An extensive quantitative study by Barash et al. (1989) has shown that in most cases the best directions of the 3 responses are similar for single area LIP and $7 \mathrm{a}$ neurons.

\section{Eye position effects}

Many neurons with IS, M, or S responses also had a tonic background activity that was related to eye position. Fourteen cells had only eye-position-related activity and were not used in this analysis. These cells were interesting nonetheless because their activity varied linearly with eye position.

Figure 4 shows the activity of such a cell from area LIP in a task in which the animal was required to fixate for $2.5 \mathrm{sec}$ at 9 different eye positions arranged on a $3 \times 3$ grid with $20^{\circ}$ spacings and centered on straight ahead. As indicated in the figure, the activity of the neuron varied monotonically with increased activity for more rightward fixations. Activity varied from 25 spikes/sec to almost 100 spikes/sec over the $40^{\circ}$ range of eye positions. This cell did not have LS, M, or S activity but the eye-position-related activity was inhibited during saccades of any dircetion or Iength.

\section{Gain fields}

The effect of eye position on LS, M, and S activity was analyzed by having the animal perform the delayed saccade task for different directions at different eye positions. Figure 5 shows the effects of eye position on direction tuning for the saccade response. The peak of the direction tuning curve does not change with eye position; only the magnitude of the response is affected. The same observation was made for the LS and M responses. In all, 56 neurons were examined at more than one direction and all showed results consistent with those illustrated in Figure 5. These results confirm previous studies of the effects of eye position on the visual responses of area 7 a neurons which showed that the visual fields remained retinotopic while the magnitude of the activity varied with eye position (Andersen et al., 1985b; Andersen and Zipser, 1988).

By examining delayed saccades made in the same direction from several different initial eye positions, we were able to map the cells' gain fields - the change in the magnitude of the LS, M, and $S$ responses corresponding to change in eye position. The direction that gave the best saccade response, determined from the direction tuning test, was used in mapping the gain field. For most cells, the LS, M, and S best directions were similar (Barash et al., 1989). Delayed saccades of the same direction and $15^{\circ}$ in amplitude were made from 9 orbital positions with $15^{\circ}$ spacings on a $3 \times 3$ grid centered on straight ahead. Figure 6 shows an example of a cell that responded best for saccades $15^{\circ}$ down. On the left are plots of the actual eye movement records for the 9 classes and on the right are the corresponding saccade responses. The saccade activity varies linearly in both the horizontal and vertical dimensions and a plane can be fit to the gain field. Planar behavior was common for the gain fields of the LS, M, and S responses for cells from areas $7 \mathrm{a}$ and LIP. These results confirm previous findings that the gain fields for the LS responses for area 7a are planar (Andersen et al., 1985b; Andersen and Zipser, 1988). Although the saccade direction and amplitude varied by small amounts with eye position, these variations had ncgligible effect on the cell's responses because the motor fields of these neurons are so large, typically averaging $90^{\circ}$ in diameter for a reduction to $50 \%$ of maximum activity (Barash et al., 1989). 
a) Saccade Direction Histograms

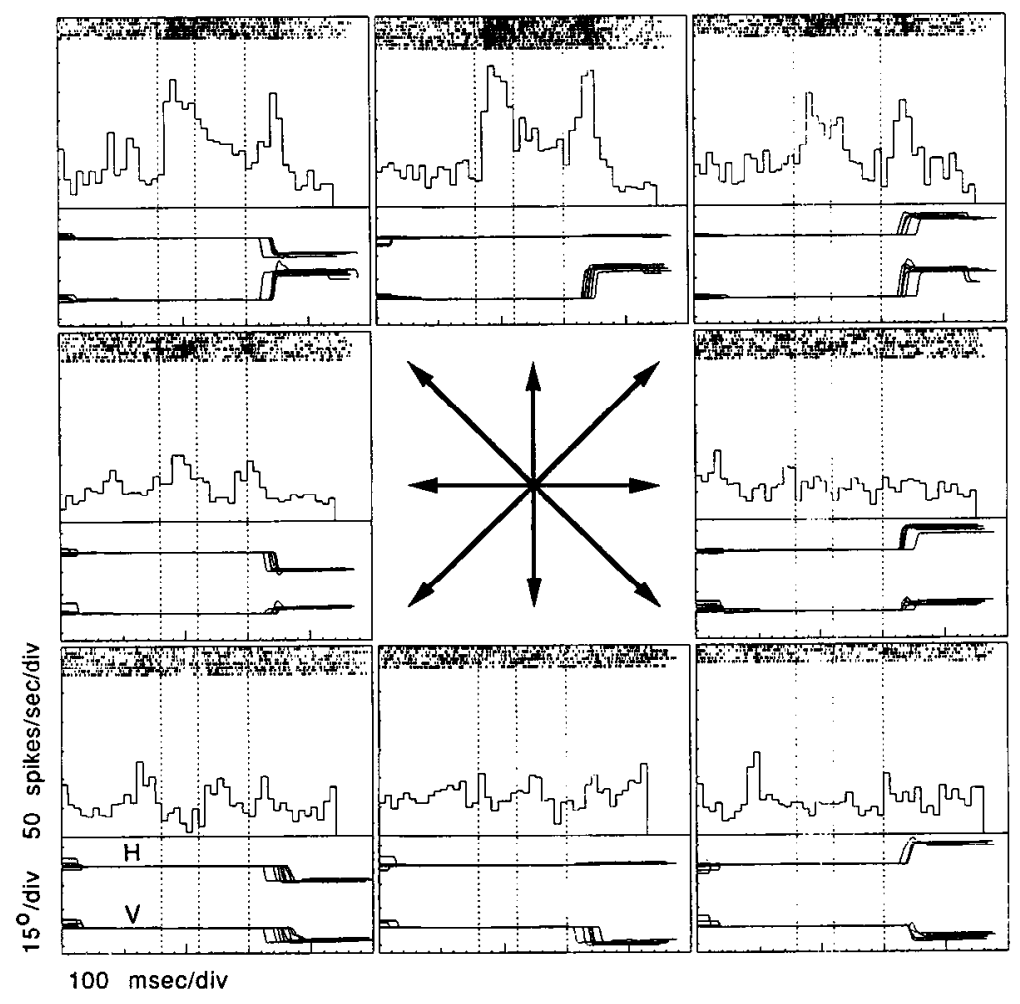

b) Light Sensitive and Saccade Polar Plots
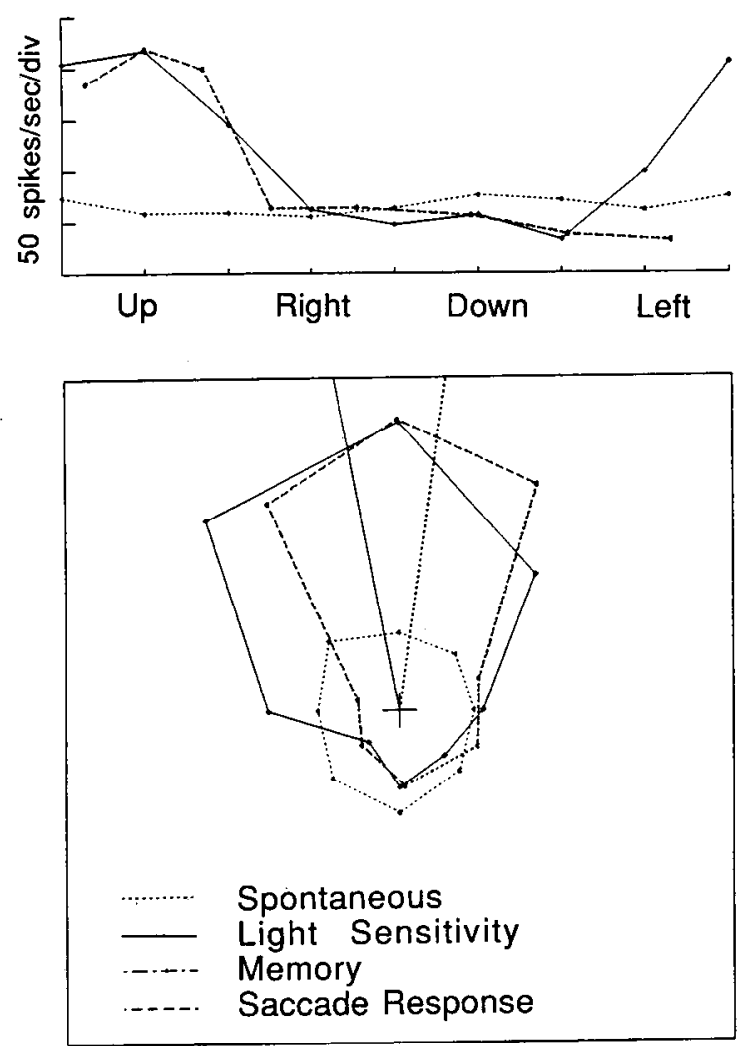

c) Light Sensitive and Memory Polar Plots
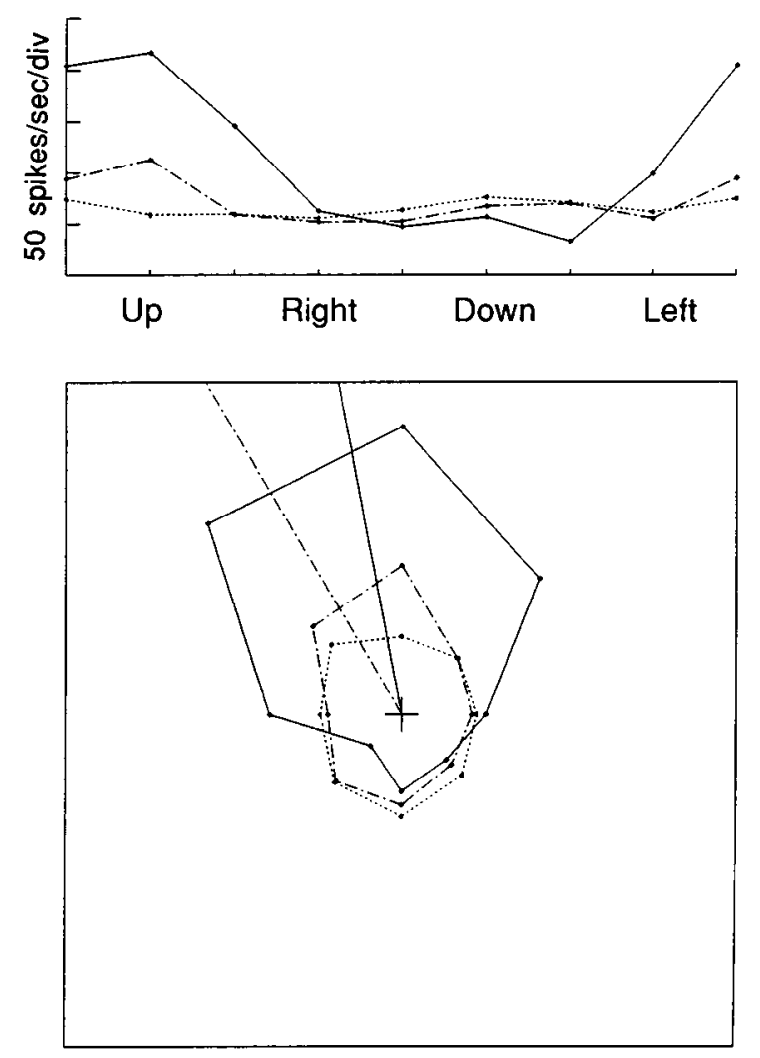
Table 1. Numbers and percentages of gain fields that fell into the four classes defined by the statistics of the two-dimensional regression analysis

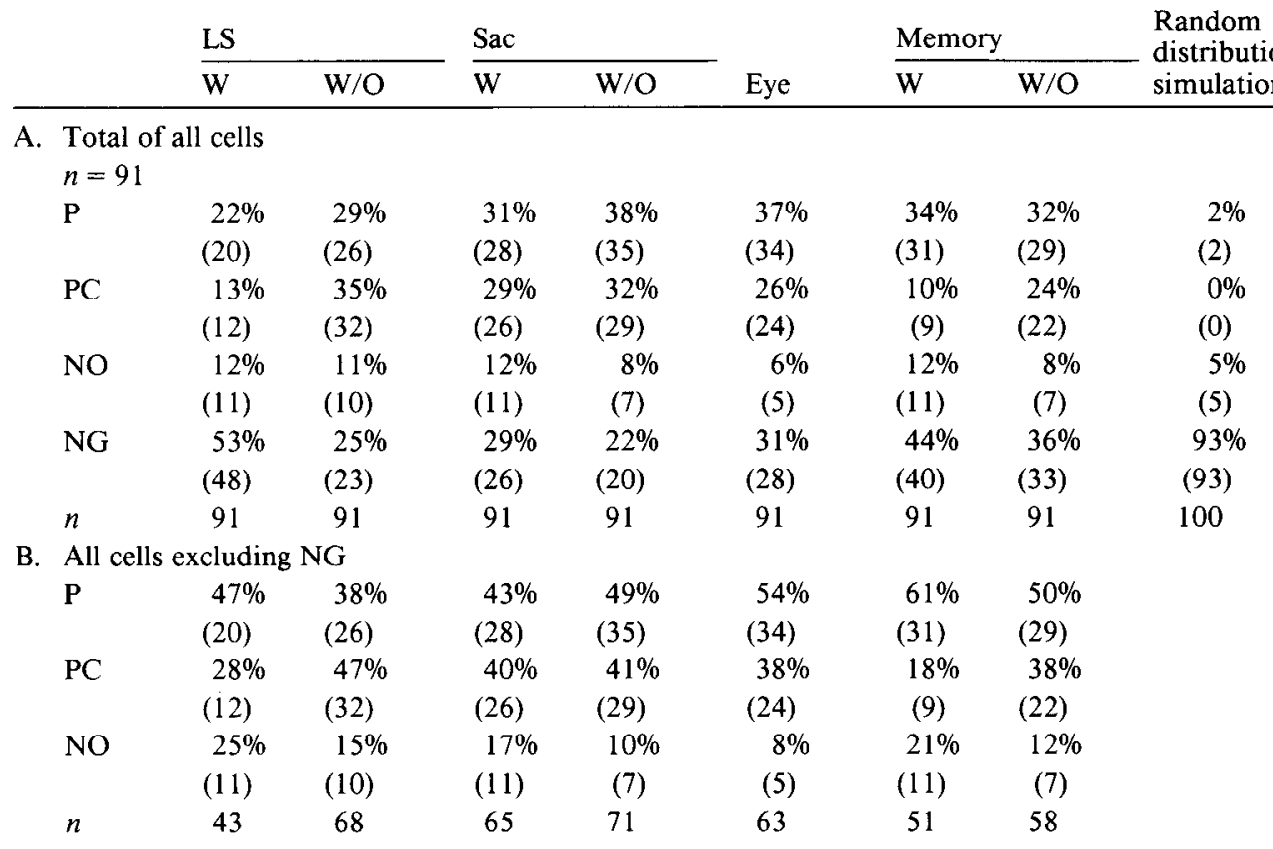

Gain fields are listed in the columns for the light-sensitive, saccade, eye position, and wait (memory) responses. W indicates that the firing rates used to compute the gain fields were "with" hackground subtraction and W/O indicates that the firing rates were "without" background subtraction. The eye position gain fields are calculated from the background rates. The rows show the percentage and number of cells in the different categories. $\mathrm{P}$, planar gain fields (regression $p<0.05$, lack-of-fit $p>0.05$ ); PC, planar component (regression $p<0.05$, lack-of-fit $p<0.05$ ); NO, no planar component (regression $p>0.05$, lack-of-fit $p<0.05$ ); $\mathrm{NG}$, no gain field (regression $p>0.05$, lack-of-fit $p>0.05$ ). The bottom row shows the total number of neurons for each column. B is the same as A, but with the percentages recomputed after taking out those cells that had no gain field (NG). The number of gain fields remaining for response class after subtracting the NG category is listed in the bottom $(n)$ row. The number of cells with NG varied by a small amount depending on whether or not background activity was subtracted, leading to different numbers within a class. For instance, 26 saccade cells with background subtraction were NG and only 20 were NG without subtraction, resulting in $n$ of 65 and 71 , respectively.

\section{Quantitative analysis of gain fields}

Linear regression techniques were used to determine if a plane was a good model for the gain fields of areas LIP and 7a. Regression analysis on the LS, $M$, and $S$ responses used 2 measures of activity. The first was a measure of the evoked response and was computed by subtracting the background activity from the total activity of the response period. For the LS and M responses the background activity value was measured at the beginning of the trial, whereas the $S$ activity was taken during the delay period just prior to the saccade. The other analysis used total activity during the test period without subtracting the background. The same basic results were recorded, using either measure, for the population of cells. Because the results were the same, only activity without background subtraction is shown in subsequent figures. We chose to use the data without background subtraction because this measurement represents the output of the cell and is the signal that it is sending to other neurons. Eye-position-related activity was also tested for the planar model by analyzing the change in background activity with eye position. The background activity for the eye position analysis was measured prior to the onset of the saccade target.

The analysis revealed 4 types of gain fields. Planar $(\mathrm{P})$ gain fields were those that had significant planar components $(p<$ $0.05)$ and no significant lack-of-fit $(p>0.05)$. For this category the planar model is the best possible fit to the data. The gain field on the left in Figure 7 is for a saccade response from a cell with a planar gain field. Planar component (PC) gain fields had significant planar components but also demonstrated a significant lack-of-fit. A portion of the variance of these gain fields could be accounted for by a plane; however, a simple linear model was not the best fit to the data. The data in the middle of Figure 7 are from a planar component gain field for a saccade response. The nonplanar (NO) gain fields had no significant

\footnotetext{
Figure 3. a, Histograms for remembered saccades made in 8 different directions. The delay period was 400 msec. The light-sensitive, memory and saccade related activities all have approximately the same best directions (up). $b$, A polar plot comparing the best-direction tuning for the lightsensitive and saccade responses. The upper graph shows the numerical values for the spontaneous, light-sensitive, and saccade activities. The lower graph is a polar plot of these values with the radius proportional to the actual activity and the direction corresponding to the direction of the visual target for the light-sensitive and spontaneous activity measures, and the actual direction of the remembered saccade for the saccade activity measure. The solid straight line indicates the calculated best direction of the light-sensitive response and the dotted line the best direction of the saccade response. $c$, Same as in $b$, but comparing the light-sensitive and memory responses.
} 


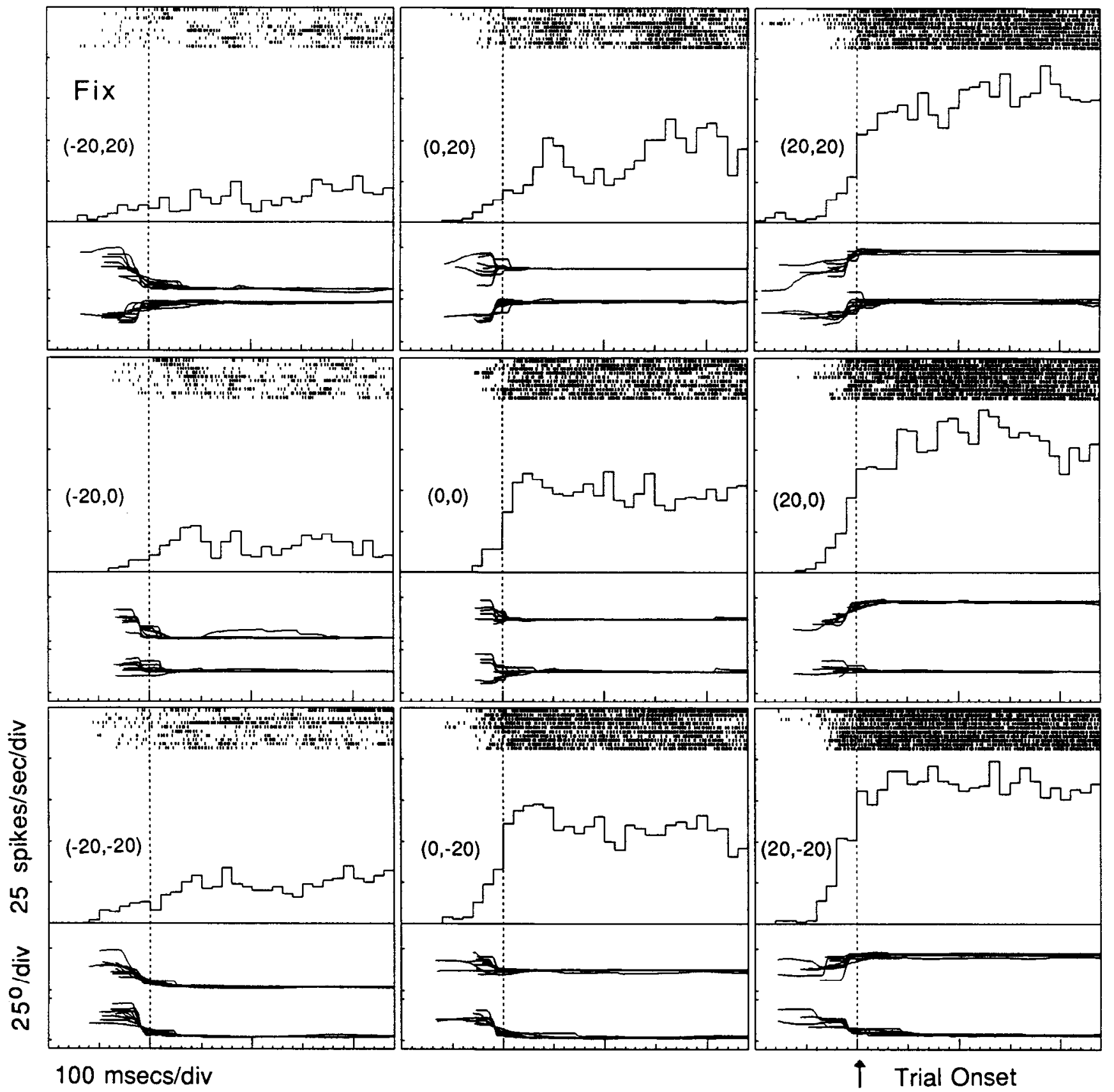

Figure 4. Eye-position-related activity for fixations at 9 different eye positions. The dotted vertical line represents the onset of the trial $100 \mathrm{msec}$ after the animal's eye position is continuously within the eye position window. Fixations are at the locations indicated by the coordinates to the left of each histogram. Note that the variation of activity with eye position approximates a plane with increased activity for more rightward fixations.

planar component but a significant lack-of-fit, indicating that while the activity did vary significantly with eye position, there was no planar component to the variation. The histograms to the right in Figure 7 illustrate a non-planar gain field for a saccade response. The fourth classification was the no gain fields (NG) which had both no significant planar component and no significant lack-of-fit; that is, there was no significant effect of eye position on the responses.

Table 1, A, shows the results of the regression analysis for all cells with and without background subtraction. The main result is that most cells that have significant gain fields for LS, M, or $S$ responses show planar or planar component behavior. From
22 to $53 \%$ of the cells showed no significant gain field, depending on the category, with an average of $34 \%$ over all categories. Table 1, B, recomputes percentages after excluding those cells with no gain fields. Most of these remaining neurons fall into the planar and planar component categories with very few in the no-planar component category. For example, for saccade responses without background subtraction, $49 \%$ of the cells showed planar gain fields and an additional $41 \%$ had planar components, with only $10 \%$ having non-planar gain fields.

An important control is to determine what percentages of $P$, $\mathrm{PC}, \mathrm{NO}$, and $\mathrm{NG}$ classes we would have encountered from a random distribution. The statistical analysis, with $p<0.05$ as 


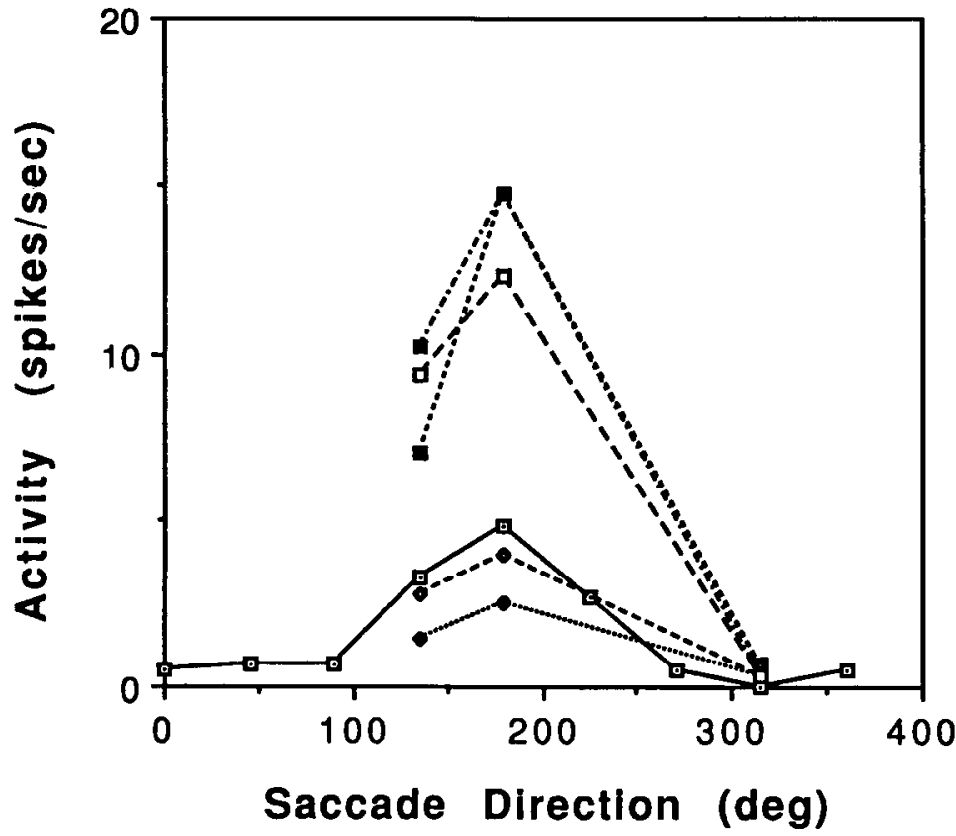

Figure 5. Direction tuning of the saccade response for saccades made from 6 different initial eye positions. Note that the magnitude of the response changes with eye position, but the best direction of the response does not. a measure of significance, would predict that $90.25 \%$ of the cclls would be NG, $4.75 \%$ would be NO, $4.75 \%$ would be $P$, and only $0.25 \%$ would be PC for a random distribution. We tested this hypothesis by generating 100 different random data files with background activity randomly distributed between 0 and 10 spikes/sec and the responses (without background subtraction) randomly distributed between 0 and $30 \mathrm{spikes} / \mathrm{sec}$. When these random data files were processed with the same analysis programs as were used on the real data, $93 \%$ were found to be NG (compared to 90.25 predicted), $5 \%$ NO (4.75 predicted),
$2 \%$ P ( $4.75 \%$ predicted), and $0 \%$ PC ( $0.25 \%$ predicted). These percentages from the random number simulation are entered in the last column of Table $1, A$, for direct comparison with the recording data.

Table 2 shows the percentages of planar, planar component, and no planar component gain field types by cortical field. Inspection of this table shows no appreciable differences between areas $7 \mathrm{a}$ and LIP.

The gradient directions for LS, M, and S activities were compared for area LIP and 7a neurons. As indicated in Figure 8 ,

Table 2. Percentages of area LIP and area 7 a cells with significant gain fields that fell into the planar, planar component, and no planar component categories

\begin{tabular}{|c|c|c|c|c|c|c|}
\hline \multirow{2}{*}{$\frac{\mathrm{LS}}{\mathrm{W}}$} & & \multicolumn{2}{|c|}{ Sac } & \multirow[b]{2}{*}{ Eye } & \multicolumn{2}{|c|}{ Memory } \\
\hline & $\mathrm{W} / \mathrm{O}$ & $\bar{W}$ & $\mathrm{~W} / \mathrm{O}$ & & $\bar{W}$ & W/O \\
\hline
\end{tabular}

A. LIP, \% all cells excluding NG$$
50 \%
$$$$
\begin{array}{cc}
41 \% & 50 \% \\
(11) & (21)
\end{array}
$$$$
P C
$$$$
30 \% \quad 36 \%
$$$$
\text { NO }
$$$$
\text { (8) }
$$$$
\text { (15) }
$$

$\begin{array}{cc}48 \% & 51 \% \\ (19) & (21) \\ 35 \% & 42 \% \\ (14) & (17) \\ 17 \% & 7 \%\end{array}$

$57 \%$

$\begin{array}{cc}68 \% & 54 \% \\ (19) & (20) \\ 21 \% & 38 \% \\ (6) & (14) \\ 11 \% & 8\end{array}$

B. $7 \mathrm{a}, \%$ all cells excluding NG

$\begin{array}{lll}\text { P } & 56 \% & 20 \% \\ & (9) & (5) \\ \text { PC } & 25 \% & 65 \% \\ & (4) & (17) \\ \text { NO } & 19 \% & 15 \% \\ & (3) & (4)\end{array}$

$n$

16

26

$\begin{array}{lc}36 \% & 47 \% \\ (9) & (14) \\ 48 \% & 40 \% \\ (12) & (12) \\ 16 \% & 13 \% \\ (4) & (4)\end{array}$
(4)

$50 \%$

(13)

$42 \%$

(11)

$8 \%$

(2)

26
(3)

(3)

37

$28 \quad 37$

$25 \quad 30$

\footnotetext{
The numbers of each type of gain field are listed in the bottom row. $W$ indicates gain fields calculated from firing rates "with" background subtraction and W/O indicates gain fields calculated from firing rates "without" background subtraction. " $A$ " lists percentages for area LIP and " $B$ " lists percentages for area 7a. There do not appear to be any significant differences between areas LIP and 7a in the types (P, PC, NO) of gain fields for the lightsensitive (LS), saccade (Sac), eye position (Eye), or memory (Wait) responses.
} 


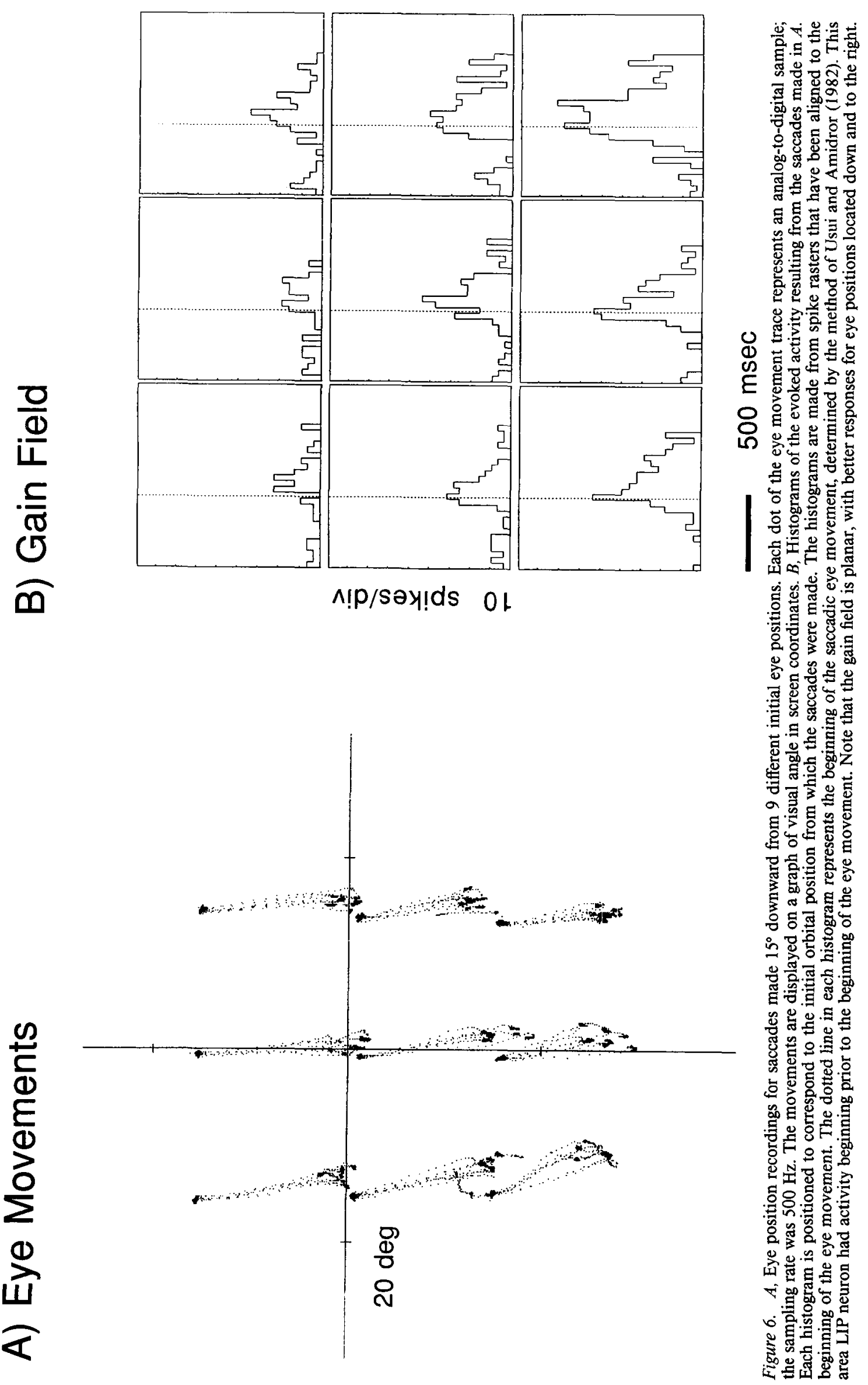


Regression $\quad p<.05$

Lack-of-fit $\quad p>.05$

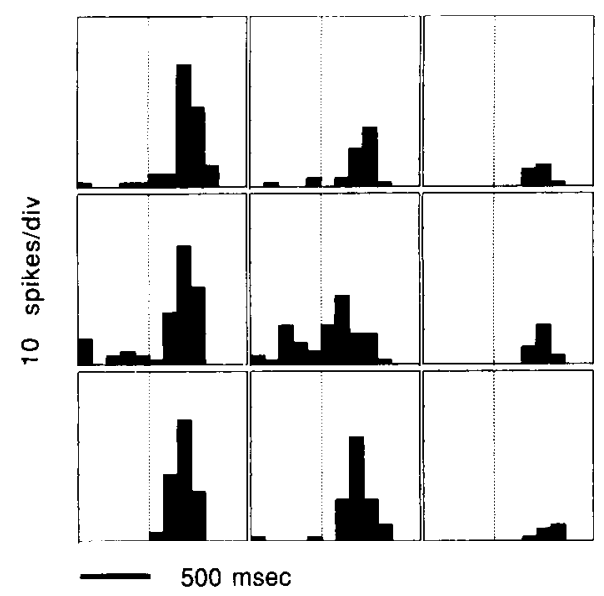

Plane

SAC

$49 \%$

LS

$38 \%$

$50 \%$ $p<.05$

$p<.05$

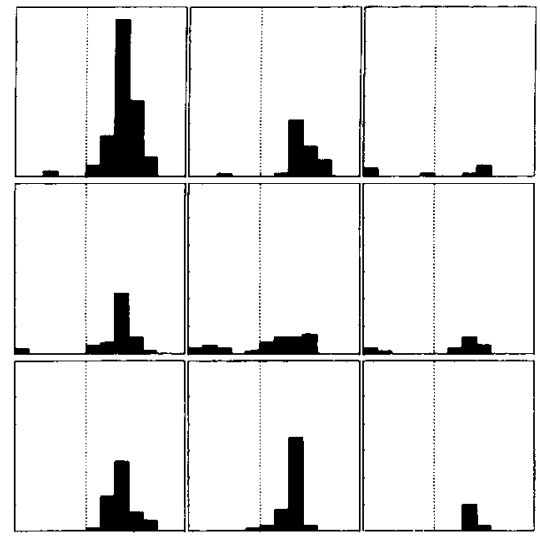

Planar Component

$41 \%$

$47 \%$

$38 \%$ $p>.05$

$p<.05$

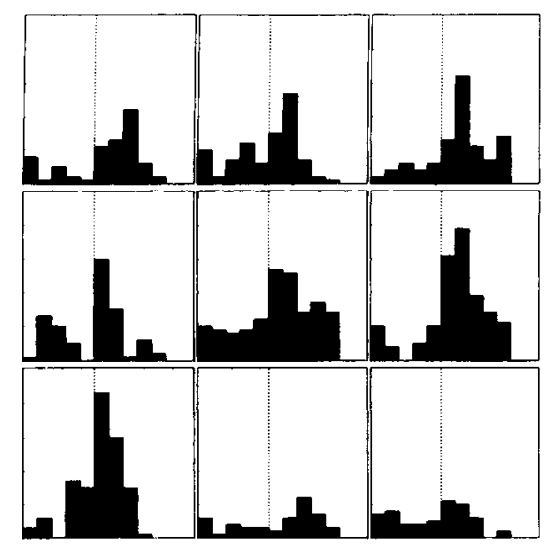

No Plane

$10 \%$

$15 \%$

$12 \%$

Figure 7. Examples of planar (left panel), planar component (middle panel), and non-planar (right panel) saccade gain fields. The histograms have not had the background activity subtracted. The dotted vertical line for each histogram indicates the time of onset of the saccade, determined by the method of Usui and Amidror, and all spike rasters that form the histograms were synchronized to the beginning of the eye movement. Planar gain fields have significant regressions $(p<0.05)$ with no significant lack-of-fit $(p>0.05)$, planar component gain fields have significant regressions with significant lack-of-fit, and non-planar gain fields have no significant regressions but significant lack-of-fit. Below each gain field example is the percentage of cells that have planar, planar component or non-planar gain fields for the saccade, light sensitive and memory responses. These percentages were computed using firing rates without background subtraction.

all 3 types of responses produced gain fields with gradients fairly evenly distributed in all directions. There did not appear to be a bias toward the contralateral or ipsilateral visual ficld. Also, no appreciable difference was noted between areas LIP and 7a.

The slopes and intercepts of the gain fields (calculated without background subtraction) are illustrated in Figures 9 and 10 and the means, medians, and other statistics are listed in Table 3. In general, the intercepts were larger for area LIP (Fig. 9), as well as the slopes (Fig. 10), indicating a larger response for area LIP. Although the background activity is also higher in area LIP (Barash et al., 1989), it accounted for only a portion of the difference.

At first glance, the intercept and slope figures appear low, but this is because the firing rate is averaged over a period of time. Often the response rate will peak at over 100 spikes/sec, but these peaks are usually quite transient, and the averaged rate, which includes the time of the peak, is usually much lower. The trial-to-trial variation is generally much smaller than the average rate of firing (Andersen et al., 1985b), indicating that these rate measures are highly reproducible from trial to trial.

Using the data from Table 3, a quantitative measure of the overall effect of eye position on the LS, M, and $S$ responses was made. The minimum and maximum activities along the horizontal and vertical axes of the gain fields were calculated by multiplying the mean slope by $15^{\circ}$ and subtracting and adding it to the mean intercept, respectively. A measure of percentage modulation was calculated as $100(1-\min / \max )$ : a value of $50 \%$ would mean that the activity varied by $50 \%$ of the maximum activity over an eye position range of $30^{\circ}$. Modulation values varied over a range of $30-47 \%$ and are shown in Table 3 , B. The modulation values were slightly higher for area $7 \mathrm{a}$, possibly because the background rate was higher in LIP and these modulation values were calculated without background subtraction. The overall average modulation was $42 \%$.

\section{Covariation of $L S, M$, and $S$ responses for single cells}

For single cells, the light-sensitive, memory, and saccade responses usually varied together as a function of eye position. Figure 11 shows an area LIP cell that gives a large response in both the LS and $S$ periods when the animal fixated left and up, and a relatively smaller response for both when the animal fixated to the right and up.

Figure 12 shows entire planar gain fields for the light-sensitive, saccade and memory responses of a single neuron. The inner- 
a)
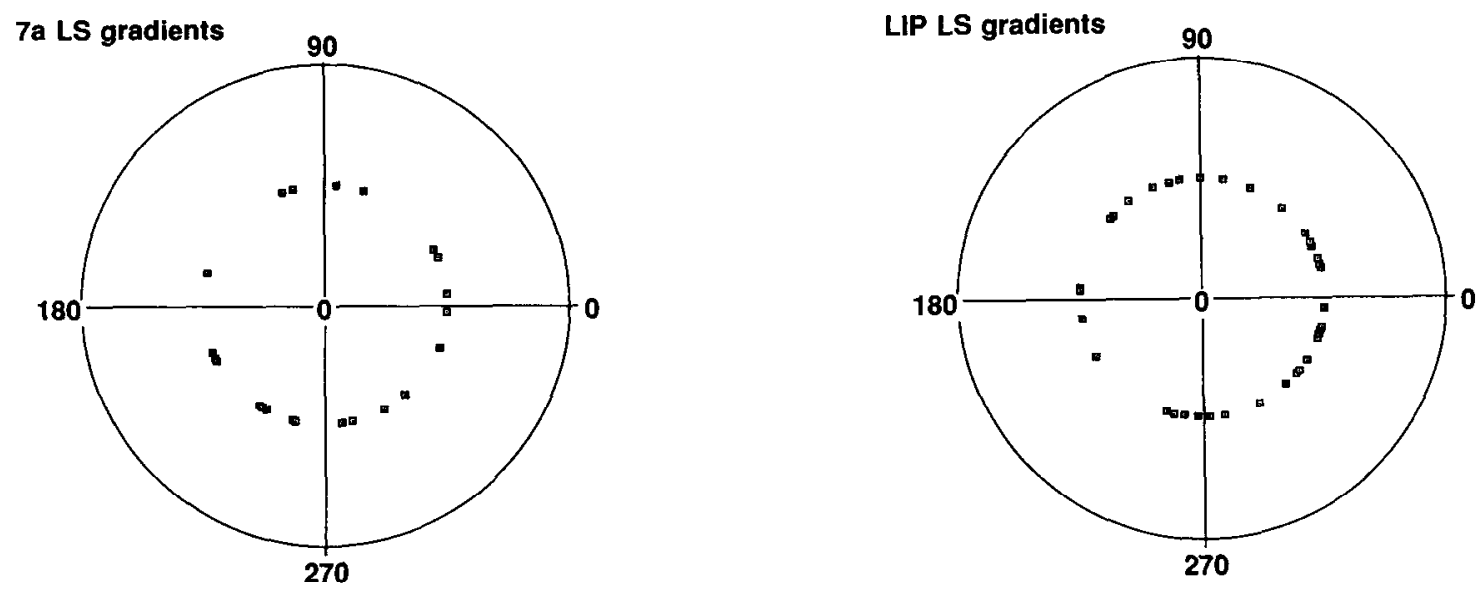

b)
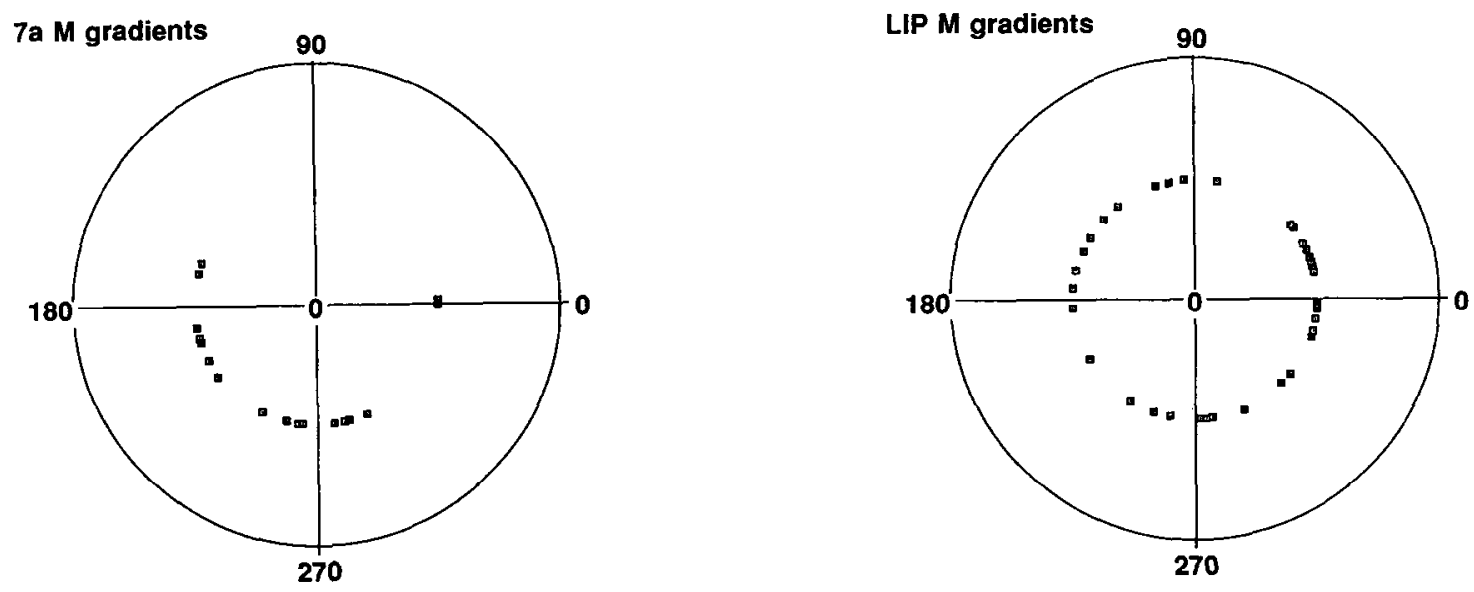

c)
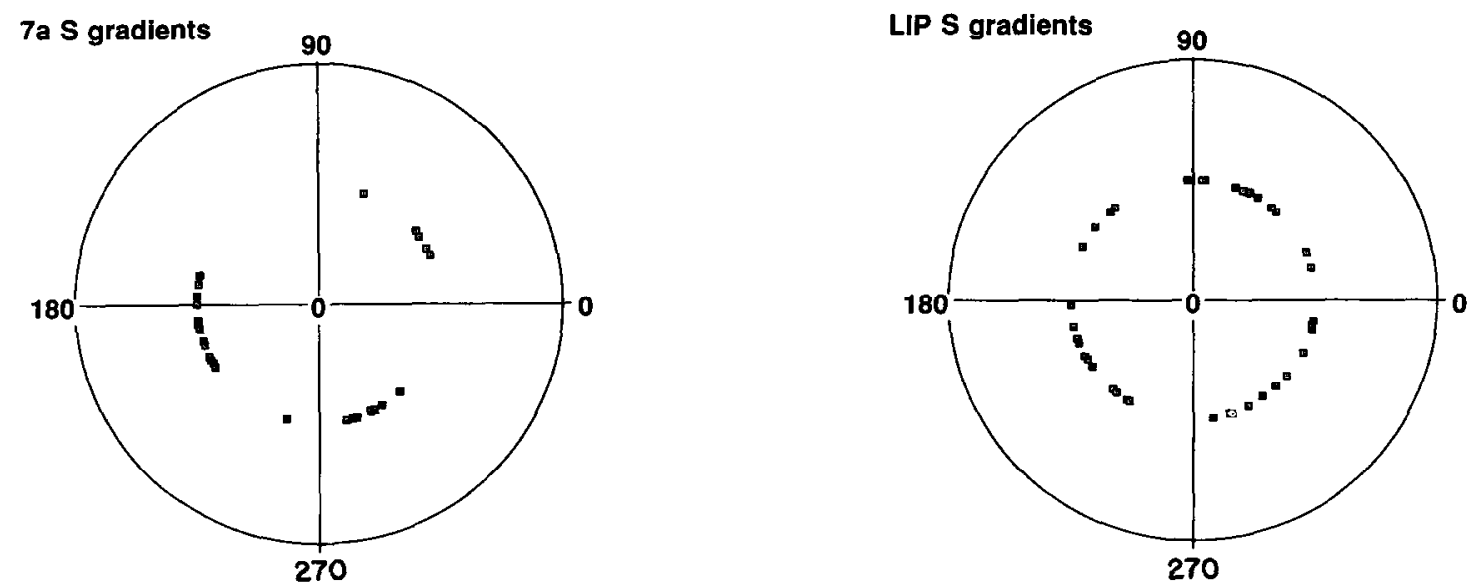

Figure 8. Direction of the gain field gradients for the light sensitivc (a), memory $(b)$, and saccade (c) gain fields for area 7a and area LIP neurons. Contralateral is to the right. Note that there is a fairly even distribution of directions for all 3 types of gain fields in both areas. The gradients were derived from gain fields using fring rates without background subtraction. Plots are from cells listed in Table 2. 
a)

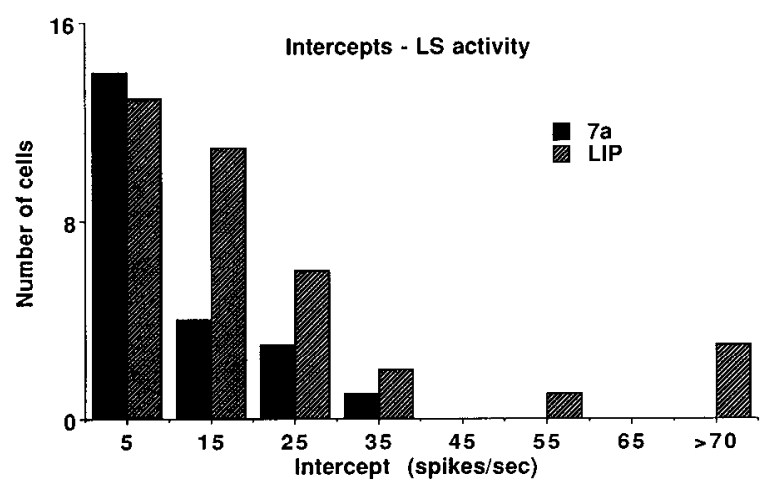

b)

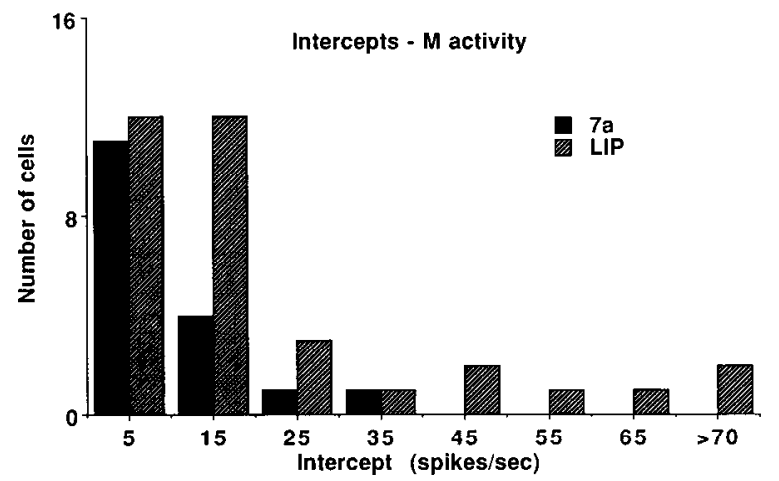

c)

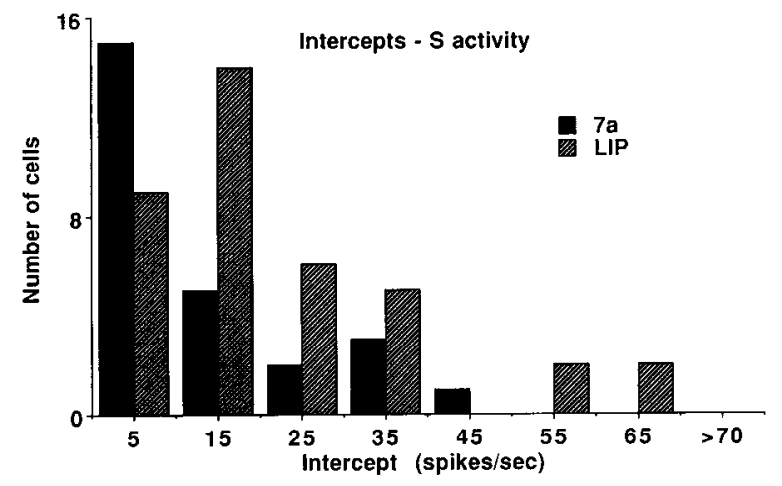

Figure 9. Intercepts for light sensitive $(a)$, memory $(b)$, and saccade (c) gain fields for area 7a and area LIP neurons. The intercepts were calculated from gain fields using firing rates without background subtraction. Note that the area LIP neurons have larger intercepts overall compared to area 7a neurons. The medians, means, standard deviations, and standard errors for the data in these histograms are listed in Table 3 .

circle diameters are proportional to the evoked activity and the outer-circle diameters to the total activity. Thus, the annuli widths are proportional to the component of activity contributed by the eye position input. Note that all 3 planes have similar gradients, i.e., directions of steepest positive slope.

The histograms in Figure 13 display the differences in the gradient directions for the light sensitive and saccade (a), lightsensitive and memory (b), and memory and saccade (c) re-
Table 3. Statistical data on intercepts and slopes of LS, M, and S gain fields and percent modulation of LS, M, and $S$ activities

\begin{tabular}{lcccll} 
& Median & Mean & SD & \multicolumn{1}{l}{ SE } & $n$ \\
\hline A. Intercepts and slopes & & & & & \\
Intercepts & & & & & \\
7a LS lateral & 8.37 & 11.296 & 8.758 & 1.867 & 22 \\
7a M lateral & 7.17 & 8.995 & 7.846 & 1.903 & 17 \\
7a S intercept & 9.04 & 13.237 & 11.184 & 2.193 & 26 \\
LIP LS & 15.40 & 25.171 & 40.276 & 6.713 & 36 \\
LIP M lateral & 13.77 & 24.263 & 32.653 & 5.6 & 34 \\
LIP S intercept & 16.39 & 21.674 & 15.963 & 2.59 & 38 \\
$X$-Slope & & & & & \\
7a LS & 0.12 & 0.188 & 0.145 & 0.037 & 15 \\
7a M & 0.16 & 0.184 & 0.146 & 0.044 & 11 \\
7a S & 0.16 & 0.200 & 0.166 & 0.035 & 22 \\
LIP LS & 0.26 & 0.294 & 0.160 & 0.033 & 23 \\
LIP M & 0.17 & 0.271 & 0.267 & 0.058 & 21 \\
LIP S & 0.24 & 0.299 & 0.225 & 0.045 & 25 \\
-Slope & & & & & \\
7a LS & 0.19 & 0.169 & 0.075 & 0.018 & 17 \\
7a M & 0.165 & 0.17 & 0.116 & 0.037 & 10 \\
7a S & 0.23 & 0.27 & 0.264 & 0.076 & 12 \\
LIP LS & 0.21 & 0.338 & 0.351 & 0.073 & 23 \\
LIP M & 0.18 & 0.285 & 0.243 & 0.053 & 21 \\
LIP S & 0.175 & 0.27 & 0.327 & 0.062 & 28 \\
B. Pex & & & & &
\end{tabular}

B. Percent modulation

$\begin{array}{lll} & X & Y \\ \text { 7a LS } & 40 \% & 37 \% \\ \text { 7a M } & 47 \% & 44 \% \\ \text { 7a S } & 37 \% & 47 \% \\ \text { LIP LS } & 30 \% & 34 \% \\ \text { LIP M } & 29 \% & 31 \% \\ \text { LIP S } & 34 \% & 31 \%\end{array}$

$A$, Statistical data on the intercepts and slopes of the LS, M, and S gain fields for areas $7 \mathrm{a}$ and LIP. All data are taken from $\mathrm{P}$ and $\mathrm{PC}$ gain fields without background subtraction listed in Table 2. The slopes listed all had significant regressions. SD, standard deviation; SE, standard error; $n$, number of gain fields. $B$. Percent modulation of the $\mathrm{LS}, \mathrm{M}$, and $\mathrm{S}$ activities over a $30^{\circ}$ range of horizontal $(X)$ or vertical $(Y)$ eye positions. The modulation was calculated as $100(1$-minimum response/maximum response).

sponses (without background subtraction) of area $7 \mathrm{a}$ and LIP neurons. Both gain fields of a comparison had to be planar or have a planar component for a cell to enter into this analysis. The differences in gradient directions were generally found to be small for all 3 comparisons for single cells. Thus, it can be concluded that the eye position input to single cells in area $7 \mathrm{a}$ and LIP produces the same effects on the magnitudes of the light-sensitive, memory, and saccade responses. Changes in eye position will increase or decrease the LS, M, and S activity together in single neurons.

\section{Discussion}

The results indicate that eye position has a significant effect on light-sensitive, memory, and saccade responses in both areas $7 \mathrm{a}$ and LIP. Fully $2 / 3$ of the cells tested showed a statistically significant effect of eye position on these responses. The direction tuning of the light-sensitive, memory, and saccade responses did not change but the magnitude of the response did. For all 3 types of responses in both areas the gain fields were usually planar or had significant planar components. The eye position 
a)

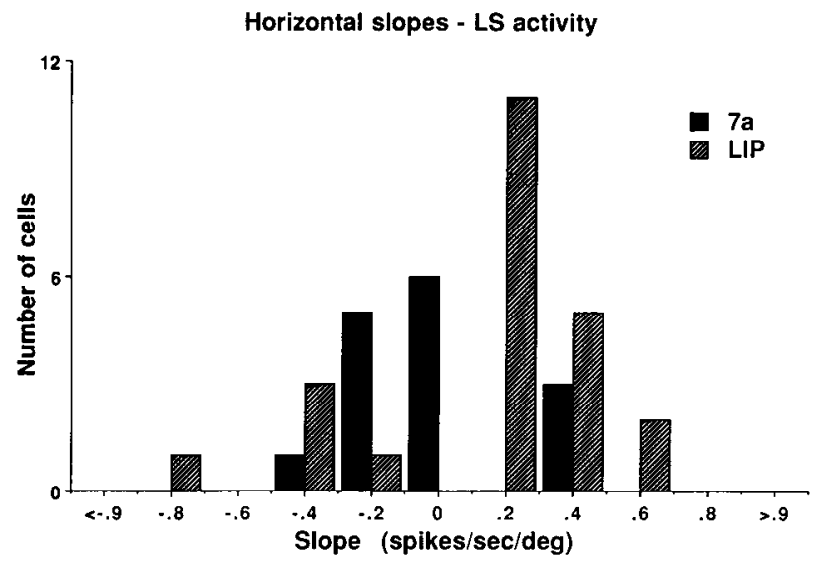

b)

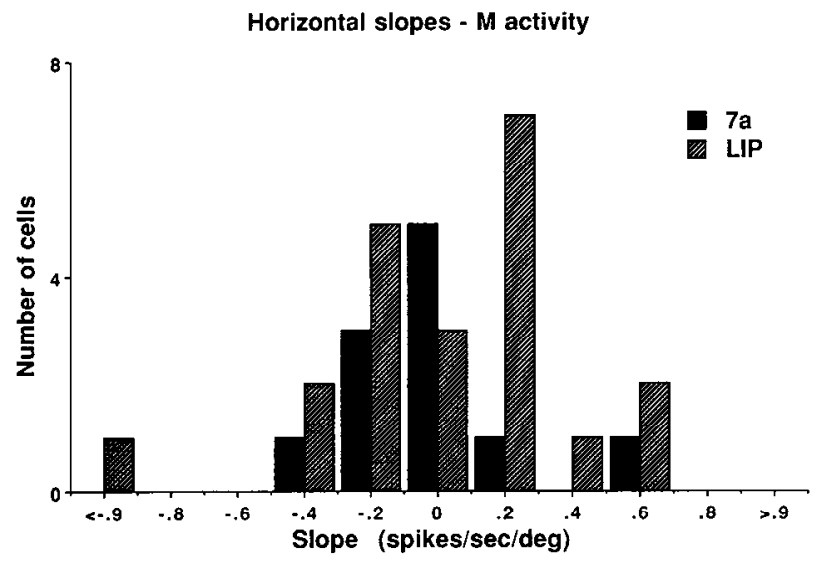

C)

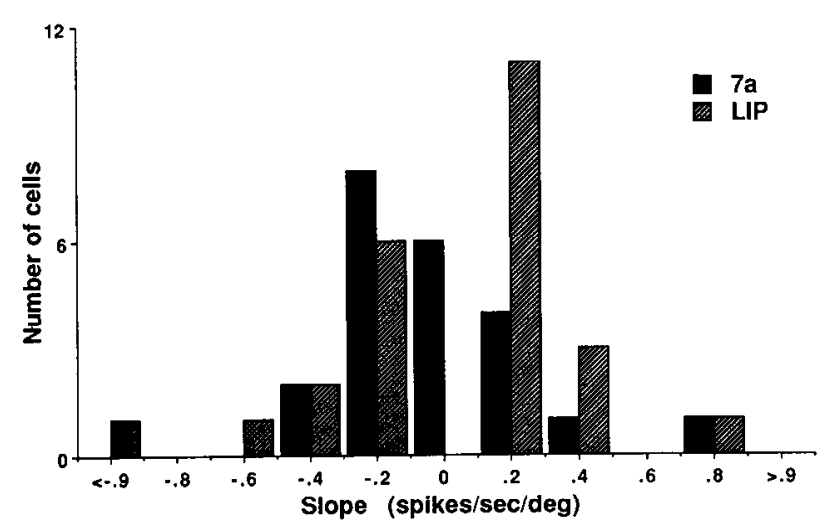

Vertical slopes - LS activity

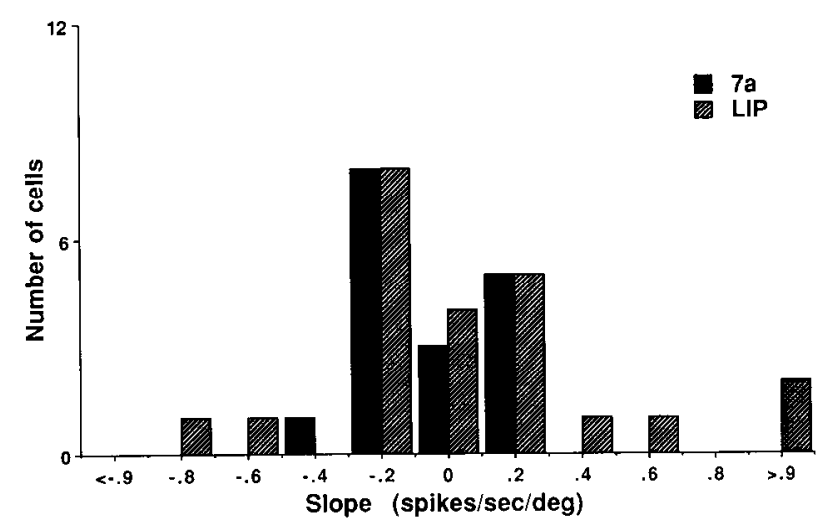

Vertical slopes - $M$ activity

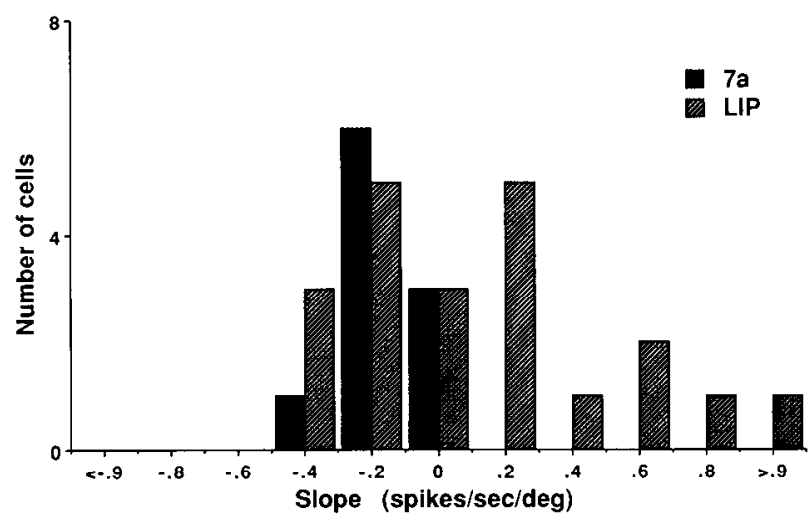

Vertical slopes - S activity

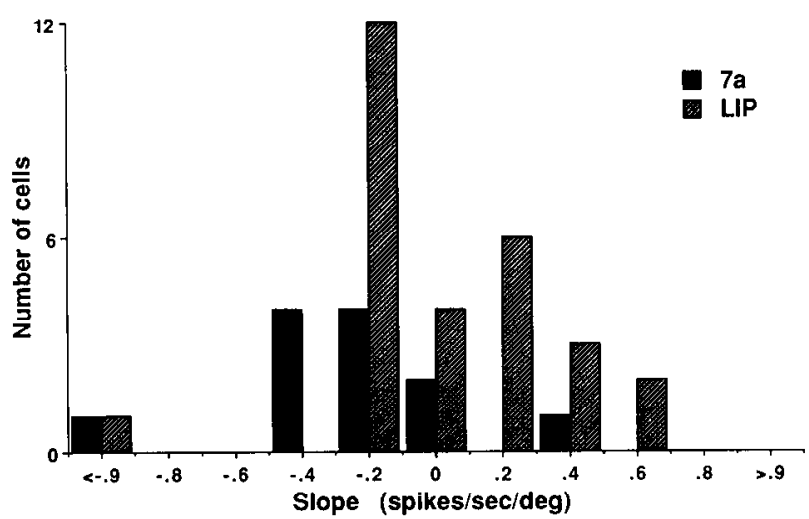

Figure 10. Horizontal and vertical slopes for the light-sensitive $(a)$, memory $(b)$, and saccadc $(c)$ gain fields for area 7 a and LIP neurons. The slopes were calculated from gain fields using firing rates without background subtraction. Only slopes that were statistically significant are plotted. The slopes for area LIP neurons are slightly larger than for area 7a neurons. The medians, means, standard deviations, and standard errors for the data in these histograms are listed in Table 3. The gain fields plotted were the same as those shown in Table 2 . The number of cells plotted are lower than in Table 2 because often a planar gain field was significant only in the vertical or only in the horizontal slope. In this figure only significant horizontal or vertical slopes are plotted. 

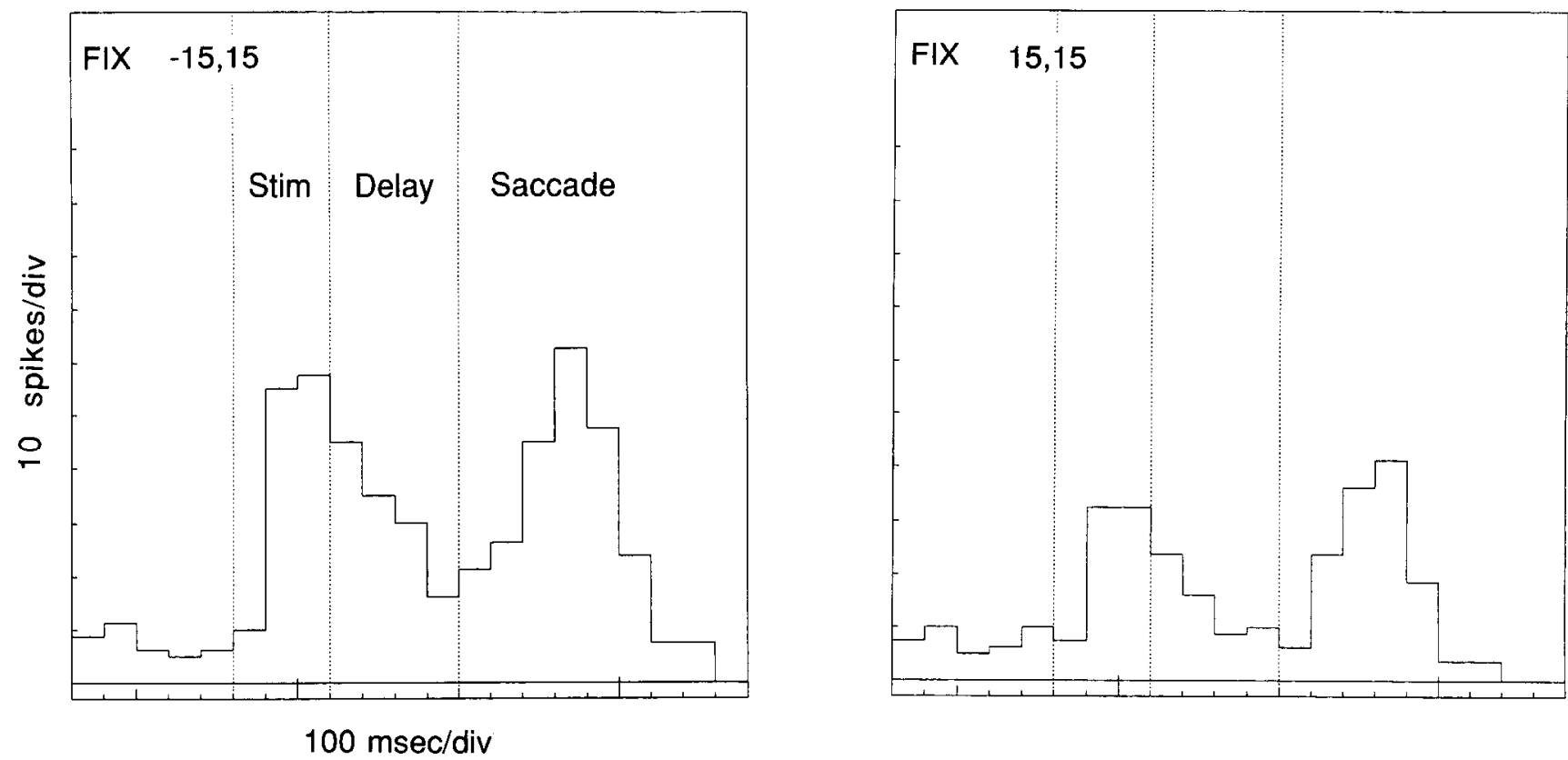

Figure 11. Responses of an area LIP neuron for eye movements made $15^{\circ}$ down and left from $(-15,15)$ initial eye position (left panel) and from $(15,15)$ initial eye position. Note that both the light-sensitive and saccade responses are larger for eye position $(-15,15)$.

signals were also usually planar. At the single cell level, the effect of eye position was usually the same for all the responses; that is, the changes in the magnitudes of the light-sensitive, memory, and saccade responses usually covaried with eye position.

Neurons in areas $7 \mathrm{a}$ and LIP had similar properties for the parameters examined in this study. Recordings were made from both cortical areas in the same animals under identical conditions, greatly strengthening the validity of the similarities. Neurons from both areas in each animal exhibited light-sensitive, memory, and saccade responses. For both areas, the best direction remained the same but the magnitude of the responses varied with eye position. Both areas showed a predominance of planar gain fields. The planar gain fields had similar gradient directions for the 2 areas. The only major difference was that area LIP intercepts and slopes were much larger, indicating that the visual, memory, and saccade responses were greater for area LIP. In a separate study using the same general population of cells from monkeys M13 and M33, Barash et al. (1989) also found that the saccade responses of area LIP neurons were presaccadic in over half of the recordings whereas the saccade responses were almost always postsaccadic for area 7 a neurons. This finding supports the conclusion of Andersen and Gnadt
(1989), who compared the latencies of saccade responses from area $7 \mathrm{a}$ of one animal with those recorded from area LIP of a different animal.

\section{Controls}

The most important control is one that rules out the possibility that the visual background could influence the response of the neurons at different eye positions. For example, at a particular eye position a contour of the test chamber might fall within the receptive field of a neuron and influence the excitability of the cell to the visual target stimulus. Likewise, during eye movements from some initial eye positions, the eye might sweep across stimuli in the background that evoke a response. To rule out these possibilities the experiments were done in total darkness, eliminating any visual background. An additional control, in which the animal made no eye movement with offset of the fixation point when no saccade target was previously presented, ruled out the possibility that the saccade response could result from the offset of the fixation target.

In earlier experiments on the effects of eye position on visual responses of area $7 \mathrm{a}$ neurons, an additional control was per-

\section{(o)}

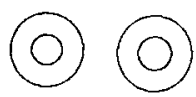

(0)
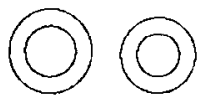

(0)

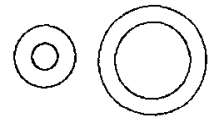

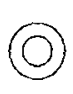
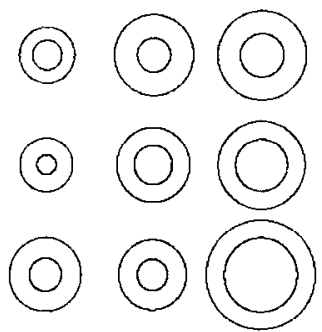
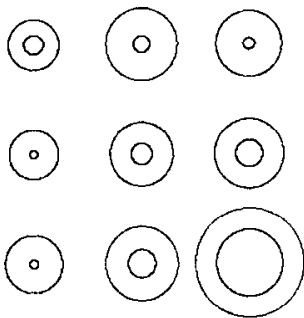

Wait
Figure 12. Light sensitive, saccade and wait (memory) gain fields for a single area LIP neuron. These data were all derived from the same memory saccade. The inner-circle diameters are proportional to the evoked activity (total activity minus background) and the outer-circle diameters are proportional to the total activity. Each pair of circles is positioned with respect to its initial eye position within the gain field. Note that the gradient directions for all three planar gain fields are very similar. 
a)

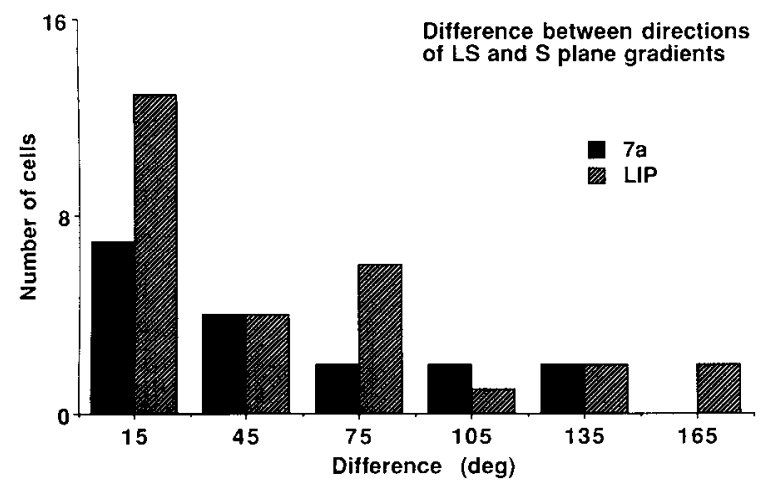

b)

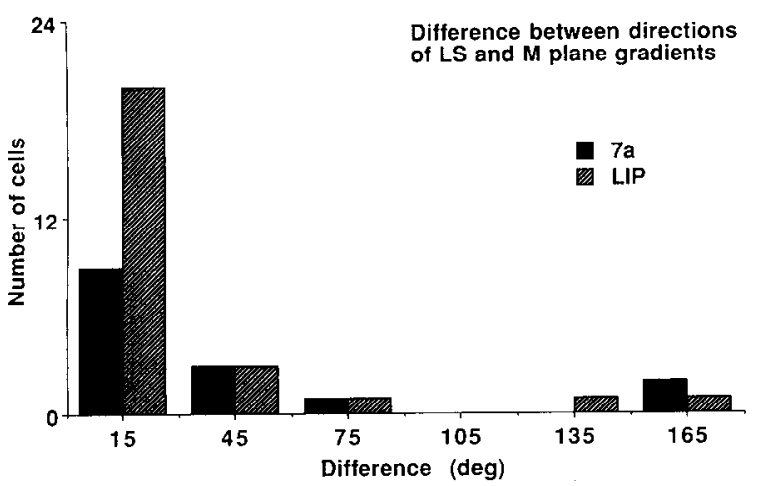

c)

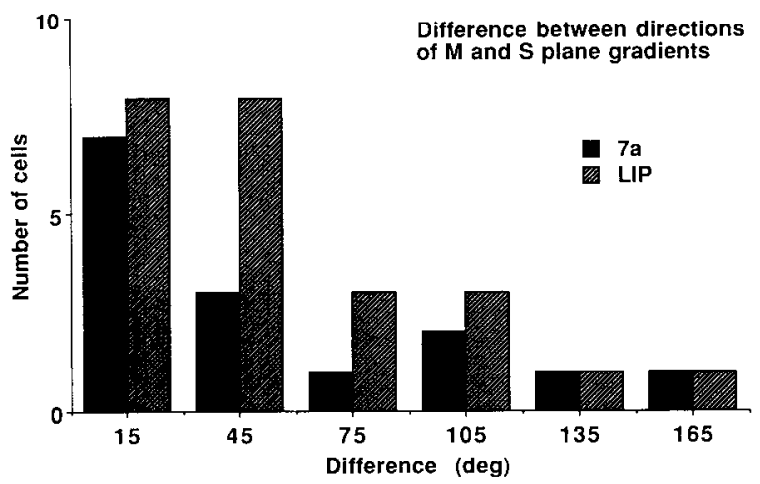

Figure 13. Differences in angle between LS and S (a), LS and $\mathbf{M}(b)$ and $M$ and $S(c)$ gradients for single area $7 a$ and LIP neurons. The gradient directions of the planar gain fields from the 3 epochs of the activity of single cells are subtracted and the absolute value of the difference is used for the histograms. Only gain fields that are planar or planar-component were analyzed. The number of cells in the histograms is smaller than that in Table 2 because both gain fields being compared in individual cells had to be statistically significant to be plotted. Note that regardless of which 2 epochs are compared, the differences tend to be small, indicating that the planar gain fields for all 3 epochs in single cells tend to have the same gradient direction.

formed in the light using prisms (Andersen et al., 1985b; Andersen and Zipser, 1988). In these experiments the monkey was required to fixate at different eye positions by looking through prisms of variable diopter values and polarity. Neither the fixation point nor the visual test stimulus was moved on the screen; rather, the animal fixated on the fixation point from different angles of gaze through the prisms. As a result, the visual back-

Desired Location of the Eyes in Head-Centered Coordinates
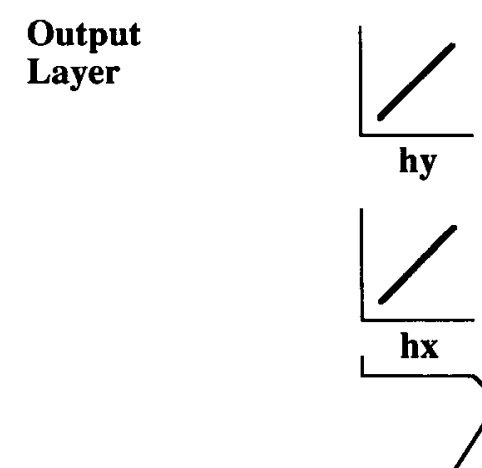

hy
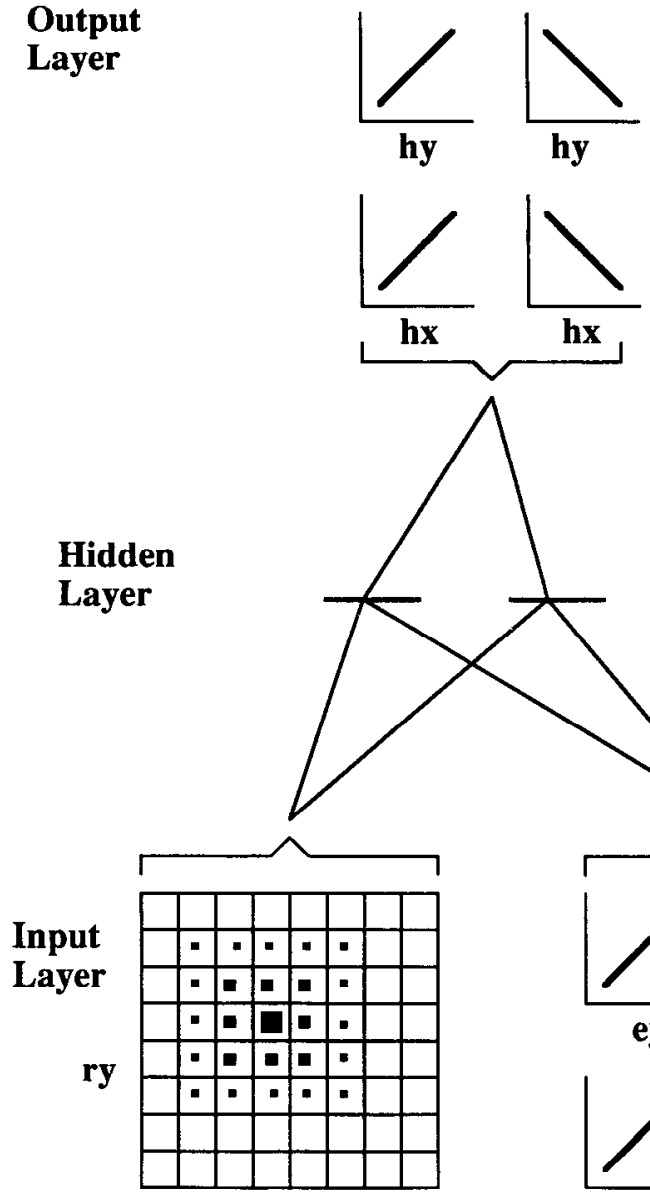

rx

\section{Retinal Position}

Figure 14. Schemalic of the neural network model trained to a linear output, adopted from Zipser and Andersen (1988). The input layer consists of an array of units coding retinal position and an array of units coding eye position. Each retinal unit has a Gaussian-shaped receptive field with a $1 / \mathrm{e}$ diameter of $15^{\circ}$. Sixty-four retinal units with centers separated by $10^{\circ}$ were used. The darkened units of the array indicate the numbers of cells stimulated by a single point of light with the diameter of each darkened area indicating the relative degree of activation. Eye position cells consisted of 4 groups whose activity varied as a linear function of eye position. Each group was comprised of 8 units and had different intercepts and slopes randomly chosen over the range of actual values recorded from eye position cells in area $7 \mathrm{a}$. Two groups werc used for horizontal eye position and 2 for vertical eye position; within each pair one group had positive slopes and the other negative slopes. Only one unit is shown from each group in the schematic. The middle or hidden layer consisted typically of 12 units that perform the coordinate transformation. Once the network is trained, it is the activity of these units that is compared to the brain recordings. The output layer contains 4 groups of 8 units that code in a linear fashion similar to the input eye position units; the difference is that instead of coding eye position, they code the location of the visual target with respect to the head. The output activities are considered to be coding the desired location of the eye in the head (in head-centered coordinates). The antagonistic (opposite slope) monotonic signals to the horizontal and vertical groups of units are a simplified approximation of the innervations that are sent to the eye muscles to move the eyes in the orbits. The horizontal activities could correspond to the innervations of the lateral and medial rectus muscles and the vertical activities to the static innervations of the vertical rectus and oblique muscles operating in a yoked fashion (Nakayama, 1975). 
ground was always retinotopically the same at the different eye positions, yet the visual response still varied with eye position.

A second potential problem is that the visual stimulus may fall on slightly different locations on the retina at different eye positions. These errors could result from errors in positioning the test stimulus, errors in fixation accuracy, or torsions of the eyes during fixation. The positions of the test stimuli were calculated and adjusted under computer control to eliminate tangent errors that would normally result from using a flat screen. Errors due to improper fixation or torsion were small, usually under $1^{\circ}$ of visual angle under the present conditions. The direction tuning of the receptive fields and motor fields of the neurons in this sample were very broad, averaging 82 and $100^{\circ}$ for a $50 \%$ reduction in activity for visual responses in areas 7 a and LIP, respectively, and 86 and $105^{\circ}$ for the motor fields of 7a and LIP neurons (Barash et al., 1988, 1989). There is a smooth variation of sensitivity between neighboring points in the receptive and motor fields and thus errors of $1^{\circ}$ would have little effect on the response of the cells. Moreover, when the entire visual receptive field was mapped at best and worst eye positions for area $7 \mathrm{a}$ neurons, it was often found that at the worst eye position the cell was completely unresponsive, regardless of where in the visual field the stimulus was presented (see figure 6 in Andersen and Zipser, 1988).

Because a tangent screen was used, we were concerned that stimuli would be presented at slightly different depths depending on the screen location of the stimulus and the point of fixation. It could be argued that small disparities or small differences in vergence could account for these results. In fact, Sakata et al. (1983) have found effects of depth on the eye position activity which suggest that the cells are coding fixation position in 3 dimensions. However, the tuning with depth was very broad in their experiments, typically varying continuously from 10 to $160 \mathrm{~cm}$. The small differences in vergence due to the use of a tangent screen would have a negligible effect according to these data. Andersen and Mountcastle (1983) compared responses with the animal fixating at different angles of gaze in which the fixation depth and relative depth of the test visual stimulus were the same. They found that the angle of gaze under these conditions still had a major influence on the response of area $7 \mathrm{a}$ neurons to visual stimuli.

Another potential source of error could arise from the fact that the intensity of the stimulus varies slightly when delivered to different locations on the screen. In monkey 13 experiments, the test stimulus intensity was maintained under computer control and calibrated so that it was always of the same intensity, regardless of screen position, measured at the animal's eye. In the second monkey the intensity was not adjusted and results were similar, suggesting that any small difference in the intensity of the stimulus was not a factor.

A last possible (although unlikely) artifact is that vertical disparities may account for the effects of eye position. Vertical disparities would be small at the screen distance used in these experiments. More important, there were large eye position effects for fixations along the vertical midline, where vertical disparities would not be a factor (see figure 11 in Andersen and Mountcastle, 1983).

It is important to note that only saccade responses were tested and as a result we refer to the activity as saccade-related. We did not test for other motor responses such as an arm or head movement toward the visual stimulus. It is possible that these cells would fire for those movements as well and may not be specific for saccades. Whether or not the responses are specific for saccades has interesting implications for the final coordinate frame of the transformation. If these cells are coding for eye and head movements, then they would be coding the "gaze location" with respect to the body (i.e., body-centered coordinates) and this location could be achieved by either an eye or head movement. We have trained network models in which head position is added to the input along with eye and retinal position and the network output is trained to locations in bodycentered coordinates (Goodman and Andersen, personal communication). We find the individual hidden units of the trained network have planar gain fields with nearly identical slope for both varying head position, holding eye and retinal position constant, and varying eye position, holding head and retinal position constant. It would be interesting to see if area LIP cells respond to head movements and, if they do, to see if the "head" gain field slopes are similar to the "eye" gain field slopes.

\section{Relation to previous studies}

Modulation of visual responses by cyc position was first rcported in the intralaminar nuclei of the thalamus (Schlag et al., 1980) and superior colliculus of the cat (Peck et al., 1980), although the nature of the modulation was not determined. Andersen and Mountcastle (1983) showed a modulation of the visual responses of area 7 a cells by eye position. This study reported effects for both stationary stimuli and stimuli moving at a constant velocity. Andersen et al. $(1984,1985 b)$ were the first to show that the visual receptive fields of area 7 a neurons remained retinotopic but the magnitude of the response was gated by eye position. These studies also first demonstrated the planar gain fields for the visual responses of area $7 \mathrm{a}$ neurons. Gating of visual responses by eye position has also been observed in monkey prefrontal cortex (Funahashi et al., 1985). In a recent study (Galletti and Battaglini, 1989) in area V3A a similar modulation of visual responses by eye position was found, including the presence of planar gain fields. Since area V3A provides input to area LIP (Andersen et al., 1989), it is possible that the eye position effects are initially produced in this area or an area even earlier in extrastriate visual cortex, although it is also possible that they are generated independently in several brain regions. Yin and Mountcastle (1978) and Mann et al. (1988) have noted a modulation of saccade responses from 2 eye positions in arca $7 \mathrm{a}$ and the supplementary eye fields of monkey, respectively. From these previous results and from the present results, it can be concluded that modulation of visual and saccadic signals by eye position is quite common in the primate and cat brain. In cases where this modulation has been examined carefully, the receptive and motor fields are always found to remain in retinotopic coordinates, but the magnitude of the response is modified by eye position. Furthermore, the effect of eye position has been found to be generally linear for both horizontal and vertical positions, producing planar gain fields. These results suggest that the method of interaction of eye and retinal position described here may be common to those regions of the brain that combine these signals. If the interactions of these signals are used for coordinate transformations from retinal to heador body-centered coordinates, then the results also suggest that the same algorithm is being used for coordinate transformations in several brain arcas.

A second line of evidence argues for the generality of this method of coordinate transformation. It has long been recognized that the processing of visually guided saccades requires 
the transformation of the retinal coordinates of the target to a signal specifying the desired location of the eyes in head-centered coordinates (Robinson, 1972, 1975; Westheimer, 1973). Investigators have looked for the brain areas in which the conversion from retinal to head-centered coordinates takes place by stimulating different saccade centers electrically through microelectrodes. It was reasoned that areas coding locations in retinal coordinates would produce saccades of equal amplitude and direction regardless of initial eye position. It was also thought that stimulating areas coding in head-centered coordinates would always drive the eye to a single "goal" in head-centered space. Thus, the direction and amplitude of the stimulus-evoked saccade would vary at different initial eye positions in order to obtain this spatial "goal."

Stimulation of many saccade centers including the superior colliculus of cat (Roucoux et al., 1976; Guitton et al., 1980; Roucoux and Crommelinck, 1980, Mcllwain, 1986) and peripheral field representation of the superior colliculus of monkey (Seagraves and Goldberg, 1984), area V1 of cat (Mcllwain, 1988), the supplementary motor field of monkey (Schlag and SchlagRey, 1987; Mann et al., 1988), the posterior parietal cortex of monkey (Shibutani et al., 1984), and cerebellum of monkey (Noda et al., 1988) produces saccades that vary their direction and amplitude as a function of eye position. Interestingly, the responses are rarely found to be "goal directed," i.e., going to a single location in head-centered coordinates regardless of initial eye position. Microstimulation of the brain at different cyc positions generally produces a convergence of eye movements toward a single location in the far peripheral head-centered space without reversal in eye movement direction. Simulated microstimulation of a neural network model of coordinate transformation in the posterior parietal cortex (Zipser and Andersen, 1988 ), outlined below, produces the same eye movement behavior (Goodman and Andersen, 1989). Since the neural network model shows retinal and eye position interactions similar to those recorded in the present study, the microstimulation results also suggest that a similar coding exists in many parts of the brain.

\section{A model for coordinate transformation}

Since the brain likely uses representations of visual space in head- and body-centered coordinates, one might expect to find areas of the brain where cells respond to a location in space regardless of eye position. However, such cells have never been found. Rather than coding in head-centered coordinates, the receptive fields of area $7 \mathrm{a}$ and LIP neurons remain retinotopic but show a modulation at response level dependent on eye position. Could such an interaction be the basis for representing visual space in head-centered coordinates?

A recent computational study suggests that the answer to this question is yes. A neural network was trained to locate positions in head-centered coordinates given eye position and retinal inputs like those found in area 7a. The hidden units of the trained model exhibited planar gain fields and modulation of the responsiveness of retinotopic receptive fields strikingly similar to the experimental results for area 7a (Andersen and Zipser, 1988; Zipser and Andersen, 1988).

Although the original model was conceived with the idea that the output representations were representing sensory space, the novel suggestion we make here is that the network could also provide the appropriate coordinate transformations for making a saccade. The original model mapped to 2 output representa- tions of location in head-centered coordinates. In the linear or monotonic representation, output units coded location of the target as a linear function of position in head-centered coordinates. The Gaussian output units coded location in a Gaussianshaped receptive field format, but with the receptive fields coding location in head-centered rather than retinal coordinates.

We wish to focus on the monotonic output model for the processing of saccades. An example of the monotonic model is shown in Figure 14. The model has 3 layers. The inputs to the model are conveyed by the first layer and contain the retinal and eye position information. The middle of "hidden" unit layer performs the coordinate transformation. In the context of the present study we wish to highlight the fact that the output representation in the monotonic model is very much like a simplified version of the static innervations that go to the extraocular muscles. The outputs contain 2 pairs of reciprocal activities, one pair coding horizontal location in head-centered space and that can be considered similar to the innervations to the lateral and medial rectus muscles and one pair coding vertical location and similar to the innervations to the vertical rectus and oblique muscles acting in a coupled fashion (Nakayama, 1975).

It is interesting to consider what the linear ouput model can accomplish in terms of modelling visual-motor integration. The model achieves the coordinate transformations for making saccadic eye movements by transforming visual targets in retinal coordinates to the locations of the eyes in the orbits in headcentered coordinates. This network also performs the conversion from a signal coded spatially in terms of a receptive field to a frequency of firing code that is appropriate for controlling muscles. Thus, the network also achieves a transformation in signal format from spatial to temporal coding, an important problem in oculomotor research (Van Gisbergen and Van Opstal, 1989).

The recording data from areas $7 \mathrm{a}$ and LIP reported here are strikingly similar to all the major features of the trained model: (1) The receptive fields are large in both areas $7 \mathrm{a}$ and LIP with respect to other extrastriate visual cortical areas. (2) The receptive fields remain retinotopic and the responses vary with eye position. (3) The gain fields are planar. (4) The receptive fields and motor fields overlap. The motor fields were determined by looking at the projection fields of the hidden units onto the output layer in the study of Goodman and Andersen (1989). They found the receptive and projection fields in the model to coincide and thus be similar to the overlap in saccade and visual receptive fields reported by Barash et al. (1989) and also illustrated in Figure 3. (5) Stimulation of the model and of areas LIP and 7a produce similar patterns of eye movements from different initial eye positions (Shibutani et al., 1984; Goodman and Andersen, 1989).

The similarities of the model and the present recording data suggest that areas 7a and I.IP are involved in processing coordinate transformations. The model shows one way in which the brain might combine the type of retinal eye information we have documented here in order to transform the retinal coordinates of visual stimuli to head-centered coordinates.

\section{Distributed coding}

The results of this study show that the direction tuning of visual, memory and saccade responses in 2 cortical fields of the posterior parietal cortex are not altered significantly by eye position but the magnitude of the response is. The degree of modulation 
of these responses is usually linear for changes in both horizontal and vertical eye position. The fact that single posterior parietal cells never have receptive fields for restricted locations in headcentered space independent of eye position, nor do the stimulation studies show convergence to a single location in headcentered space, suggests that the code for spatial location is distributed. The neural network model helps us to understand how such a distributed coding could be used. Since, in the model, the only explicit coding of single locations in space independent of eye position is found in the sum of the outputs, and this output is interpreted to be the signal for desired locations of the eyes in the orbits, the coding for spatial location could, in theory, remain distributed throughout the visual and oculomotor systems and only be explicit in the behavior of moving the eyes. The distributed nature of the coding suggests that single neurons with receptive fields for small regions of head-centered or bodycentered space may not exist, and searching for such neurons may prove to be futile.

\section{References}

Andersen, R. A. (1987) The role of the inferior parietal lobule in spatial perception and visual-motor integration. In The Handbook of Physiology. Section 1: The Nervous System. Volume IV. Higher Functions of the Brain. Part 2, F. Plum, V.B. Mountcaste, and S.T. Geiger, eds., pp. 483-518, American Physiological Society, Bethesda, MD.

Andersen, R. A., and J. Gnadt (1989) Role of posterior parietal cortex in saccadic eye movements. In Reviews in Oculomotor Research, Vol. III, R. Wirtz and M. Goldberg, eds., pp. 315-335, Elsevier, Amsterdam.

Andersen, R. A., and V. B. Mountcastle (1983) The influence of the angle of gaze upon the excitability of the light-sensitive neurons of the posterior parietal cortex. J. Neurosci. 3: 532-548.

Andersen, R. A., and D. Zipser (1988) The role of the posterior parietal cortex in coordinate transformations for visual-motor integration. Can. J. Physiol. Pharmacol. 66: 488-501.

Andersen, R. A., G. K. Essick, and R. M. Siegel (1984) The role of eye position on the visual response of neurons in area 7a. Neurosci. Abstr. 10: 934 .

Andersen, R. A., C. Asanuma, and W. M. Cowan (1985a) Callosal and prefrontal associational projecting cell populations in area $7 \mathrm{a}$ of the macaque monkey: A study using retrogradely transported fluorescent dyes. J. Comp. Neurol. 232: 443-455.

Andersen, R. A., G. K. Essick, and R. M. Siegel (1985b) Encoding of spatial location by posterior parietal neurons. Science 230: 456-458.

Andersen, R. A., G. K. Essick, and R. M. Siegel (1987) Neurons of area 7 activated by both visual stimuli and oculomotor behavior. Exp. Brain Res. 67: 316-322.

Andersen, R. A., C. Asanuma, G. Essick, and R. M. Siegel (1989) Cortico-cortical connections of anatomically and physiologically defined subdivision within the inferior parietal lobule. J. Comp. Neurol. (in press).

Barash, S., R. Andersen, M. Bracewell, J. Gnadt, and L. Fogassi (1988) Saccade-related activity in area LIP. Neurosci. Abstr. 14: 203.

Barash, S., R. M. Bracewell, L. Fogassi, and R. A. Andersen (1989) Interactions of visual and motor-planning activities in the lateral intra-parietal area (LIP). Neurosci. Abstr. 15: 1203.

Blatt, G. J., G. R. Stoner, and R. A. Andersen (1987) The lateral intraparietal area (LIP) in the macaque: associational connections and visual receptive field organization. Neurosci. Abstr. 13: 627.

Blatt, G., R. A. Andersen, and G. Stoner (1989) Visual receptive field organization and LIP cortico-cortical connections of area LIP in the macaque. J. Comp. Neurol. (in press).

Bruce, C. J., and M. E. Goldberg (1985) Primate frontal eye fields. I. Single neurons discharging before saccades. J. Neurophysiol. 53:603635 .

Fuchs, A. F., and D. A. Robinson (1966) A method for measuring horizontal and vertical eye movement chronically in the monkey. $J$. Appl. Physiol. 21: 1068-1070.

Funahashi, S., C. J. Bruce, and P. S. Goldman-Rakic (1985) Visual properties of prefrontal cortical neurons. Neurosci. Abstr. 11: 525.
Galletti, C., and P. P. Battaglini (1989) Gaze-dependent visual neurons in area $\mathrm{V} 3 \mathrm{~A}$ of monkey prestriatc cortcx. J. Neurosci. 9: 1112-1125.

Gallyas, F. (1979) Silver staining of myelin by means of physical development. Neuorol. Res. 1: 203-209.

Gnadt, J. W., and R. A. Andersen (1986) Spatial, memory, and motorplanning properties of saccade-related activity in the lateral intraparietal area (LIP) of macaque. Neurosci. Abstr. 13: 454.

Gnadt, J. W., and R. A. Andersen (1988) Memory related motor planning activity in posterior parietal cortex of macaque. Exp. Brain Res. 70: 216-220.

Gnadt, J. W., R. M. Bracewell, and R. A. Andersen (1987) Saccadic eye movements without visual feedback: Memory-linked sensorimotor spatial transformation. Neurosci. Abstr. 13: 1090.

Gnadt, J., M. Bracewell, and R. A. Andersen (1989) Characteristics of eye movements to remembered visual targets. Vision Res. (in press).

Goldman-Rakic, P. S. (1988) Topography of cognition: Parallel distributed networks in primate association cortex. Annu. Rev. Neurosci. 11: 137-156.

Goodman, S. J., and R. A. Andersen (1989) Microstimulation of a neural-network model for visually guided saccades. J. Cognitive Neurosci. $1:$ 317-326.

Guitton, D., M. Crommelinck, and A. Roucoux (1980) Stimulation of the superior colliculus in the alert cat. I. Eye movement and neck EMG activity evoked when the head is restrained. Exp. Brain Res. 39: 63-73.

Judge, S. J., B. J. Richmond, and F. C. Chu (1980) Implantation of magnetic search coils for measurement of eye position: an improved method. Vision Res. 43: 1133.

Kleinbaum, D. G., and L. L. Kupper (1978) Applied Regression Analysis and Other Multivariable Methods, Duxbury, North Scituate, MA.

Lynch, J. C., V. B. Mountcastle, W. H. Talbot, and T. C. T. Yin (1977) Parietal lobe mechanisms of directed visual attention. J. Neurophysiol. $40: 362-389$.

Mann, S. E., R. Thau, and P. H. Schiller (1988) Conditional taskrelated responses in monkey and dorsomedial frontal cortex. Exp. Brain Res. 69: 460-468.

Mays, L. E., and D. L. Sparks (1980) Dissociation of visual and saccade-related responses in superior colliculus neurons. J. Neurophysiol, 43: 207-231.

McIlwain, J. T. (1986) Effects of eye position on saccades evoked electrically from superior colliculus of alert cats. J. Neurophysiol. 55 . 97-112.

McIlwain, J. T. (1988) Saccadic eye movements evoked by electrical stimulation of the cat's visual cortex. Visual Neurosci. 1: 135-143.

Nakayama, K. (1975) Coordination of extraocular muscles. In Basic Mechanisms of Ocular Motility and Their Clinical Implications, G. Lemnerstrand and P. Bach-y-Rita, eds., pp. 193-207, Perganon, New York.

Netter, J., and W. Wasserman (1983) Applied Linear Regression Analysis, Irwin, Homewood, IL.

Noda, H., S. Murakami, J. Yamada, Y. Tamaki, and T. Aso (1988) Saccadic eye movements evoked by microstimulation of the fastigial nucleus of macaque monkeys. J. Neurosci. 60: 1036-1052.

Peck, C. K., M. Schlag-Rey, and J. Schlag (1980) Visuo-oculomotor properties of cells in the superior colliculus of the alert cat. J. Comp. Neurol. 194: 97-116.

Robinson, D. A. (1972) Eye movements evoked by collicular stimulation in the alert monkey. Vision Res. 12:1795-1808.

Robinson, D. A. (1975) Oculomotor controls signals. Part III. Are saccades retinotopically or spatially organized? In Basic Mechanisms of Ocular Motility and Their Clinical Implications, G. Lennerstrand and P. Bach-y-Rita, eds., pp. 366-374, Pergamon, New York.

Roucoux, A., and M. Crommelinck (1980) Stimulation of the superior colliculus in the alert cat. II. Eye and head movements evoked when the head is unrestrained. Exp. Brain Res. 39: 75-85.

Roucoux, A., D. Guitton, and M. Crommenlinck (1976) Eye movements evoked by superior colliculus stimulation in the alert cat. Brain Res. 106: 349-363.

Sakata, H., H. Shibutani, and K. Kawano (1983) Functional properties of visual tracking neurons in posterior parietal association cortex of the monkey. J. Neurophysiol. 49: 1364-1380.

Schlag, J., and M. Schlag-Rey (1987) Evidence for a supplementary eye field. J. Neurophysiol. 57: 179-200.

Schlag, J., M. Schlag-Rey, C. K. Peck, and J.-P. Joseph (1980) Visual responses of thalamic neurons depending on the direction of gaze and the position of targets in space. Exp. Brain Res. 40: 170-184. 
Seagraves, M. A., and M. E. Goldberg (1984) Initial orbital position affects the trajectories of large saccades evoked by electrical stimulation of the monkey superior colliculus. Soc. Neurosci. Abstr. 10: 59.

Shibutani, H., H. Sakata, and J. Hyvarinen (1984) Saccade and blinking evoked by microstimulation of the posterior parietal association cortex of the monkey. Exp. Brain Res. 55: 1-8.

Ungerleider, L. G., and R. Desimone (1986) Cortical connections of visual area MT in the macaque. J. Comp. Neurol. 248: 190-222.

Usui, S., and I. Amidror (1982) Digital low-pass differentiation for biological signal processing. IEEE Trans. Biomed. Eng. BME-29:686693.

Van Gisbergen, J. A. M., and A. J. Van Opstal (1989) Saccadic eye movements as a control system. Chapter 3. Models. In The Neurobiology of Saccadic Eye Movements, R. H. Wurtz and M. E. Goldberg, eds., Elsevier, Amsterdam.

Westheimer, G. (1973) Saccadic eye movements. In The Oculomotor System and Brain Functions, Publishing House of Slovak Academy of Sciences, Bratislava, Czechoslovakia.

Yin, T. C., and V. B. Mountcastle (1978) Mechanisms of neural integration in the parietal lobe for visual attention. Fed. Proc. 37: 22512257.

Zipser, D., and R. A. Andersen (1988) A back-propagation programmed network that simulated response properties of a subset of posterior parietal neurons. Nature 331: 697-684. 